TRANSACTIONS OF THE

AMERICAN MATHEMATICAL SOCIETY

Volume 360, Number 7, July 2008, Pages 3687-3730

S 0002-9947(08)04371-7

Article electronically published on January 25, 2008

\title{
STEIN'S METHOD AND RANDOM CHARACTER RATIOS
}

\author{
JASON FULMAN
}

\begin{abstract}
Stein's method is used to prove limit theorems for random character ratios. Tools are developed for four types of structures: finite groups, Gelfand pairs, twisted Gelfand pairs, and association schemes. As one example an error term is obtained for a central limit theorem of Kerov on the spectrum of the Cayley graph of the symmetric group generated by $i$-cycles. Other main examples include an error term for a central limit theorem of Ivanov on character ratios of random projective representations of the symmetric group, and a new central limit theorem for the spectrum of certain random walks on perfect matchings. The results are obtained with very little information: a character formula for a single representation close to the trivial representation and estimates on two step transition probabilities of a random walk. The limit theorems stated in this paper are for normal approximation, but many of the tools developed are applicable for arbitrary distributional approximation.
\end{abstract}

\section{INTRODUCTION}

Given a fixed $m \times m$ matrix, it is natural to study the distribution of its eigenvalues, where each eigenvalue is chosen with probability $\frac{1}{m}$. As a motivating example, consider the $n ! \times n$ ! transition matrix for random walk on the symmetric group $S_{n}$, where the generating set consists of all $i$-cycles. Diaconis and Shahshahani [10] proved that the eigenvalues of this matrix are the numbers $\frac{\chi_{(i, 1 n-i)}^{\lambda}}{\operatorname{dim}(\lambda)}$ occurring with multiplicity $\operatorname{dim}(\lambda)^{2}$. Here $\lambda$ parameterizes an irreducible representation of the symmetric group, $\chi_{\left(i, 1^{n-i}\right)}^{\lambda}$ is the corresponding character value on $i$-cycles, and $\operatorname{dim}(\lambda)$ is the dimension of the irreducible representation. Since $\sum_{|\lambda|=n} \operatorname{dim}(\lambda)^{2}=n$ !, the eigenvalue $\frac{\chi_{\left(i, 1^{n-i}\right)}^{\lambda}}{\operatorname{dim}(\lambda)}$ is chosen with probability $\frac{\operatorname{dim}(\lambda)^{2}}{n !}$.

The probability measure on irreducible representations of $S_{n}$ which picks the representation corresponding to $\lambda$ with probability $\frac{\operatorname{dim}(\lambda)^{2}}{n !}$ is known as the Plancherel measure of the symmetric group. Kerov [26] proved that if $i \geq 2$ is fixed, and $\lambda$ is random from the Plancherel measure of the symmetric group, then the random variable $\frac{\left.\sqrt{\left(\begin{array}{l}n \\ i\end{array}\right)(i-1) !} \chi_{(i, 1}^{\lambda} \lim ^{n-i}\right)}{\operatorname{dim}(\lambda)}$ is asymptotically normal as $n \rightarrow \infty$. Kerov used the method of moments and difficult combinatorics; a beautiful exposition of his work is the paper 24. Hora 21 gave another proof of Kerov's result, also using the method of moments, but with somewhat simpler combinatorics. In very recent work, Sniady

Received by the editors August 16, 2005, and in revised form, May 13, 2006.

2000 Mathematics Subject Classification. Primary 05E10; Secondary 60 C05.

Key words and phrases. Stein's method, normal approximation, Gelfand pair, character ratio, symmetric group, Plancherel measure, association scheme.

The author was supported by NSA grant H98230-05-1-0031 and NSF grant DMS-0503901.

(C)2008 American Mathematical Society

Reverts to public domain 28 years from publication 
40. uses the genus expansion of random matrix theory to give another method of moments proof of Kerov's result.

A more probabilistic approach to Kerov's result for the case $i=2$ was given in [14, where Stein's method was used to obtain the first error term in Kerov's central limit theorem; an error term of $O\left(n^{-1 / 4}\right)$ was proved and an error term of $O\left(n^{-1 / 2}\right)$ was conjectured. This error term was later improved to $O\left(n^{-s}\right)$ for any $s<1 / 2$ using martingale theory [17]. More recently, a proof of the $O\left(n^{-1 / 2}\right)$ conjecture appears in 39, using a new refinement of Stein's method. See [16] for another proof of the $O\left(n^{-1 / 2}\right)$ bound, using Bolthausen's variation of Stein's method. All of these results, it should be emphasized, were only for the case $i=2$. However even in the simple setting of $i=2$, random character ratios arise in work on the moduli space of curves 12 .

Before proceeding further, it should be mentioned that familiarity with Stein's method is not necessary to read this paper. Section 2 gives a brief introduction to normal approximation by Stein's method. It presents the bare minimum needed to understand this paper, but gives a few pointers to the literature for further reading.

Section 3 of this paper generalizes the set-up of Kerov's central limit theorem to the case when $G$ is a finite group and the generating set consists of a single conjugacy class $C$. This elucidates our early work on this problem [14] which required different information than the current treatment. As a new application, it is shown that for any fixed $i \geq 2$, one obtains an error term $O\left(n^{-1 / 4}\right)$ in Kerov's central limit theorem. The approach presented here uses only the most elementary ingredients, namely a well known character formula for the irreducible representation parameterized by $\lambda=(n-1,1)$ on all elements of $S_{n}$, and estimates on the two step transition probabilities of the random walk on $S_{n}$ generated by $C$.

Section 4 uses Stein's method to study random spherical functions of a Gelfand pair. As an application, a new central limit theorem with error term $O\left(n^{-1 / 4}\right)$ is obtained for the spectrum of certain random walks on the set of perfect matchings of $2 n$ symbols. Equivalently, a central limit theorem is obtained for certain statistics under the $\mathrm{Jack}_{2}$ measure on partitions, which is an interesting object [33. As in the group case, only the simplest ingredients are needed in the proof.

Section 5 focuses on a specific example of a twisted Gelfand pair. An error term is obtained for a central limit theorem of Ivanov 23] on character ratios of random projective representations of the symmetric group. There is a close parallel to earlier sections, but new ideas are required since if one tries to generalize the approach used in the treatment of Gelfand pairs, one encounters a Markov chain which can have negative transition probabilities. A main contribution of Section 5 is a combinatorial argument designed to overcome this obstacle.

Section [6] develops limit theorems for the spectrum of an adjacency matrix of an association scheme. The arguments are analogous to those in previous sections. This is not surprising since Gelfand pairs and association schemes are both generalizations of the finite group case. However the perspective and examples are quite different. We treat the Hamming association scheme, obtaining a central limit theorem for the spectrum of the Hamming graph, or equivalently for values of $q$-Krawtchouk polynomials.

Having outlined the contents of this article, we mention further reasons why the results are interesting. First, the construction of an exchangeable pair and certain moment computations are applicable for distributional approximations other than 
normal approximation. The paper [6] illustrates this in the context of exponential approximation. Second, the spectrum of random walks on $G / K$, where $(G, K)$ is a Gelfand pair, is of ongoing interest. In particular if $G, K$ are finite classical groups, this leads to difficult questions in number theory [43, 44] and we are optimistic that the exchangeable pair constructed in this paper will yield useful information. Third, Plancherel measure (which arises in the group case), Jack measure (which arises in the Gelfand pair case), and shifted Plancherel measure (which arises in the twisted Gelfand pair case), are all objects of interest to researchers in random matrix theory [1, 4], 3, 7], 25, 31, 32], 33, 45]. As is evident from these papers, there are many interesting statistics under these measures, and the method of constructing exchangeable pairs in this paper allows one to study these statistics by Stein's method. Fourth, the examples in this paper will be a useful testing ground for results on Stein's method. For example the refinement of Stein's method in [39] arose from trying to obtain an $O\left(n^{-1 / 2}\right)$ error term for the $i=2$ case of Kerov's central limit theorem. In forthcoming work we obtain an $O\left(n^{-1 / 2}\right)$ bound for Theorem 3.14 and a similar approach gives an $O\left(n^{-1 / 2}\right)$ bound for Theorems 4.19 and 5.2

\section{Stein's METHOD FOR NORMAL APPROXimation}

In this section we briefly review Stein's method for normal approximation, using the method of exchangeable pairs [41]. One can also use couplings to prove normal approximations by Stein's method (see 34] for a survey), but the exchangeable pairs approach is effective for our purposes. For a survey discussing both exchangeable pairs and couplings, the paper 36 can be consulted.

Two random variables $W, W^{\prime}$ on a state space $X$ are called exchangeable if for all $w_{1}, w_{2}, \mathbb{P}\left(W=w_{1}, W^{\prime}=w_{2}\right)$ is equal to $\mathbb{P}\left(W=w_{2}, W^{\prime}=w_{1}\right)$. As is typical in probability theory, let $\mathbb{E}(A \mid B)$ denote the expected value of $A$ given $B$. The following result of Stein (which follows from page 35 of 41 and Lemma 2.3 below) uses an exchangeable pair $\left(W, W^{\prime}\right)$ to prove a central limit theorem for $W$.

Theorem 2.1 (41]). Let $\left(W, W^{\prime}\right)$ be an exchangeable pair of real random variables such that $\mathbb{E}\left(W^{2}\right)=1$ and $\mathbb{E}\left(W^{\prime} \mid W\right)=(1-a) W$ with $0<a<1$. Then for all real $x_{0}$,

$$
\begin{aligned}
& \left|\mathbb{P}\left(W \leq x_{0}\right)-\frac{1}{\sqrt{2 \pi}} \int_{-\infty}^{x_{0}} e^{-\frac{x^{2}}{2}} d x\right| \\
\leq & \frac{\sqrt{\operatorname{Var}\left(\mathbb{E}\left[\left(W^{\prime}-W\right)^{2} \mid W\right]\right)}}{a}+(2 \pi)^{-\frac{1}{4}} \sqrt{\frac{1}{a} \mathbb{E}\left|W^{\prime}-W\right|^{3}} .
\end{aligned}
$$

In order to apply Theorem 2.1 to study a statistic $W$, one needs an exchangeable pair $\left(W, W^{\prime}\right)$. The usual way of doing this is to use Markov chain theory. A Markov chain $K$ (with the chance of going from $x$ to $y$ denoted by $K(x, y)$ ) on a finite set $X$ is called reversible with respect to a probability distribution $\pi$ if $\pi(x) K(x, y)=$ $\pi(y) K(y, x)$ for all $x, y$. This condition implies that $\pi$ is a stationary distribution for $K$. It is straightforward to check that if $K$ is reversible with respect to $\pi$, then one obtains an exchangeable pair $\left(W, W^{\prime}\right)$ as follows: choose $x \in X$ from $\pi$, then obtain $x^{\prime}$ by taking one step from $x$ according to $K$, and set $\left(W, W^{\prime}\right)=\left(W(x), W\left(x^{\prime}\right)\right)$.

A drawback with Theorem 2.1 is that in many problems of interest, it gives a convergence rate of $O\left(n^{-1 / 4}\right)$ rather than $O\left(n^{-1 / 2}\right)$. When $\left|W^{\prime}-W\right|$ is bounded, the following variation often gives the correct rate. A similar result is in [35]. 
Theorem $2.2\left([39)\right.$. Let $\left(W, W^{\prime}\right)$ be an exchangeable pair of real random variables such that $\mathbb{E}\left(W^{2}\right)=1$ and $\mathbb{E}\left(W^{\prime} \mid W\right)=(1-a) W$ with $0<a<1$. Suppose that $\left|W^{\prime}-W\right| \leq A$ for some constant $A$. Then for all real $x_{0}$,

$$
\begin{aligned}
& \left|\mathbb{P}\left(W \leq x_{0}\right)-\frac{1}{\sqrt{2 \pi}} \int_{-\infty}^{x_{0}} e^{-\frac{x^{2}}{2}} d x\right| \\
\leq & \frac{\sqrt{\operatorname{Var}\left(\mathbb{E}\left[\left(W^{\prime}-W\right)^{2} \mid W\right]\right)}}{2 a}+.41 \frac{A^{3}}{a}+1.5 A .
\end{aligned}
$$

The following general lemma will also be helpful.

Lemma 2.3. Let $\left(W, W^{\prime}\right)$ be an exchangeable pair of random variables such that $\mathbb{E}\left(W^{\prime} \mid W\right)=(1-a) W$ and $\mathbb{E}\left(W^{2}\right)=1$. Then $\mathbb{E}\left(W^{\prime}-W\right)^{2}=2 a$.

Proof. Since $W$ and $W^{\prime}$ have the same distribution,

$$
\begin{aligned}
\mathbb{E}\left(W^{\prime}-W\right)^{2} & =\mathbb{E}\left(\mathbb{E}\left(W^{\prime}-W\right)^{2} \mid W\right) \\
& =\mathbb{E}\left(\left(W^{\prime}\right)^{2}\right)+\mathbb{E}\left(W^{2}\right)-2 \mathbb{E}\left(W \mathbb{E}\left(W^{\prime} \mid W\right)\right) \\
& =2 \mathbb{E}\left(W^{2}\right)-2 \mathbb{E}\left(W \mathbb{E}\left(W^{\prime} \mid W\right)\right) \\
& =2 \mathbb{E}\left(W^{2}\right)-2(1-a) \mathbb{E}\left(W^{2}\right) \\
& =2 a .
\end{aligned}
$$

\section{Finite groups}

This section uses Stein's method to study the spectrum of random walk on a finite group $G$, where the generating set is a conjugacy class $C$ which satisfies $C=C^{-1}$. As will be explained in Subsection 3.1, by [10] this is equivalent to studying the distribution of the character ratio $\frac{\chi^{\lambda}(C)}{\operatorname{dim}(\lambda)}$ where $\lambda$ is chosen from the Plancherel measure of the group $G$.

The organization of this section is as follows. Subsection 3.1 recalls the necessary background from representation theory. Subsection 3.2 then defines a Markov chain on the set of irreducible representations of $G$, and uses it to construct an exchangeable pair. This leads to a general central limit theorem. Subsection 3.3 applies the theory to the symmetric group $S_{n}$ with $C$ the conjugacy class of $i$-cycles, where $i$ is fixed and $n$ is large.

3.1. Background from representation theory. We recall facts from the representation theory of finite groups, referring the reader to Chapter 1 of [37] or to the first few chapters of 38 for more details. In what follows, $\chi$ denotes a character of the finite group $G, \operatorname{dim}(\chi)$ is the dimension of the corresponding representation, and $\operatorname{Irr}(G)$ is the set of all irreducible characters of $G$. Also $\bar{z}$ denotes the complex conjugate of a number $z$.

Lemma 3.1. Let $\chi$ be an irreducible representation of $G$. Then $\chi\left(C^{-1}\right)=\overline{\chi(C)}$. Thus if $C=C^{-1}$, then $\chi(C)$ is real.

Next we recall the orthogonality relations for irreducible characters of $G$.

Lemma 3.2. Let $\nu$ and $\chi$ be irreducible characters of a finite group $G$. Then

$$
\frac{1}{|G|} \sum_{g \in G} \nu(g) \overline{\chi(g)}=\delta_{\nu, \chi}
$$


Lemma 3.3. Let $C$ be the conjugacy class of $G$ containing the element $g$. Then for $g \in G$,

$$
\sum_{\chi \in \operatorname{Irr}(G)} \chi(g) \overline{\chi(h)}
$$

is equal to $\frac{|G|}{|C|}$ if $h, g$ are conjugate and is 0 otherwise.

Lemma 3.4 while known, is perhaps not well known, and since analogous results will be needed in later sections, a proof along the lines of one in [19] is included.

Lemma 3.4. Let $G$ be a finite group with conjugacy classes $C_{1}, \cdots, C_{t}$. Let $C_{k}$ be the conjugacy class of an element $w \in G$. Then the number of $m$-tuples $\left(g_{1}, \cdots, g_{m}\right) \in G^{m}$ such that $g_{j} \in C_{i_{j}}$ and $g_{1} \cdots g_{m}=w$ is

$$
\prod_{j=1}^{m}\left|C_{i_{j}}\right| \sum_{\chi \in \operatorname{Irr}(G)} \frac{\operatorname{dim}(\chi)^{2}}{|G|} \frac{\chi\left(C_{i_{1}}\right)}{\operatorname{dim}(\chi)} \cdots \frac{\chi\left(C_{i_{m}}\right)}{\operatorname{dim}(\chi)} \frac{\overline{\chi\left(C_{k}\right)}}{\operatorname{dim}(\chi)} .
$$

Proof. Identify each class $C_{i}$ with its corresponding class sum in the complex group algebra $\mathbb{C} G$. If $\chi^{(1)}, \cdots, \chi^{(t)}$ are the irreducible complex characters of $G$, then the elements

$$
E_{s}=\frac{\operatorname{dim}\left(\chi^{(s)}\right)}{|G|} \sum_{j=1}^{t} \overline{\chi_{j}^{(s)}} C_{j} \quad(1 \leq s \leq t)
$$

are a complete set of orthogonal idempotents for the center of $\mathbb{C} G$, where $\chi_{j}^{(s)}$ denotes the value of $\chi^{(s)}$ at any $g \in C_{j}$. Lemma 3.3 implies that

$$
C_{j}=\left|C_{j}\right| \sum_{s=1}^{t} \frac{\chi_{j}^{(s)}}{\operatorname{dim}\left(\chi^{(s)}\right)} E_{s} .
$$

Since the $E_{j}$ 's are orthogonal idempotents (i.e. $E_{r} E_{s}=\delta_{r, s} E_{r}$ ), it follows that

$$
\begin{aligned}
C_{i_{1}} C_{i_{2}} \cdots C_{i_{m}} & =\left|C_{i_{1}}\right| \cdots\left|C_{i_{m}}\right| \sum_{s=1}^{t} \frac{\chi_{i_{1}}^{(s)} \cdots \chi_{i_{m}}^{(s)} E_{s}}{\operatorname{dim}\left(\chi^{(s)}\right)^{m}} \\
& =\frac{\left|C_{i_{1}}\right| \cdots\left|C_{i_{m}}\right|}{|G|} \sum_{k=1}^{t} C_{k} \sum_{s=1}^{t} \frac{\chi_{i_{1}}^{(s)} \cdots \chi_{i_{m}}^{(s)} \overline{\chi_{k}^{(s)}}}{\operatorname{dim}\left(\chi^{s}\right)^{m-1}},
\end{aligned}
$$

as desired.

As a corollary one obtains the following result.

Corollary 3.5 (10]). Suppose that $C$ is a conjugacy class satisfying $C=C^{-1}$. Then the eigenvalues of the random walk on $G$ with generating set $C$ are indexed by $\chi \in \operatorname{Irr}(G)$ and are the numbers $\frac{\chi(C)}{\operatorname{dim}(\chi)}$, occurring with multiplicity $\operatorname{dim}(\chi)^{2}$.

Proof. If $M$ is the $|G| \times|G|$ transition matrix for the random walk, the chance of being at the identity after $k$ steps is the trace of $M^{k}$ divided by $|G|$. Thus Lemma 3.4 implies that for all $k \geq 0$, the trace of $M^{k}$ is equal to

$$
\sum_{\chi \in \operatorname{Irr}(G)} \operatorname{dim}(\chi)^{2}\left(\frac{\chi(C)}{\operatorname{dim}(\chi)}\right)^{k}
$$

Since this holds for all $k \geq 0$, the result follows. 
As mentioned in the Introduction, the Plancherel measure of $G$ is the probability measure on $\operatorname{Irr}(G)$ which chooses each $\chi$ with probability $\frac{\operatorname{dim}(\chi)^{2}}{|G|}$. So Corollary 3.5 says that the eigenvalues of the random walk on $G$ generated by $C$ are the "character ratios" $\frac{\chi(C)}{\operatorname{dim}(\chi)}$ occurring with multiplicity proportional to the Plancherel probability of $\chi$.

3.2. Central limit theorems for character ratios. The goal of this subsection is to prove a central limit theorem for the random variable $W$ defined by $W(\lambda)=$ $\frac{|C|^{1 / 2} \chi^{\lambda}(C)}{\operatorname{dim}(\lambda)}$, where $C$ is a fixed conjugacy class such that $C=C^{-1}$ and $\lambda$ is random from the Plancherel measure of $G$. From Lemmas 3.1 and 3.3 it follows that $\mathbb{E}(W)=$ 0 if $C$ is not the identity class, and that $\mathbb{E}\left(W^{2}\right)=1$.

To apply Stein's method it is useful to construct a Markov chain on the set of irreducible representations of $G$ as follows. First, fix an irreducible representation $\tau$ whose character is real valued. This gives a Markov chain $L_{\tau}$ by defining the probability of transitioning from $\lambda$ to $\rho$ as

$$
L_{\tau}(\lambda, \rho):=\frac{\operatorname{dim}(\rho)}{\operatorname{dim}(\lambda) \operatorname{dim}(\tau)} \frac{1}{|G|} \sum_{g} \chi^{\lambda}(g) \chi^{\tau}(g) \overline{\chi^{\rho}(g)} .
$$

Let us make some remarks about the chain $L_{\tau}$. First, note that the quantity $\frac{1}{|G|} \sum_{g} \chi^{\lambda}(g) \chi^{\tau}(g) \overline{\chi^{\rho}(g)}$ is the multiplicity of $\rho$ in the tensor product of $\lambda$ and $\tau$. (For probabilist readers who might be unfamiliar with such algebraic facts, we refer to pages 37 and 50 of [37.) Hence the chance of going from $\lambda$ to $\rho$ is determined by how the tensor product of $\lambda$ and $\tau$ decomposes into irreducibles. A closely related construction was used for Stein's method in the earlier work 14. It should also be noted that the idea of using tensor products to define random walks on irreducible representations had been previously used in the context of compact Lie groups, where the state space is infinite (see for instance [13]).

Lemma 3.6 verifies that $L_{\tau}$ is a Markov chain which is reversible with respect to Plancherel measure.

Lemma 3.6. The transition probabilities of $L_{\tau}$ are real and non-negative and sum to 1. Moreover the chain $L_{\tau}$ is reversible with respect to the Plancherel measure of $G$.

Proof. The transition probabilities of $L_{\tau}$ are real and non-negative since as noted above,

$$
\frac{1}{|G|} \sum_{g} \chi^{\lambda}(g) \chi^{\tau}(g) \overline{\chi^{\rho}(g)}
$$

is the multiplicity of $\rho$ in the tensor product of $\lambda$ and $\tau$. Letting $i d$ denote the identity, it follows from Lemma 3.3 that

$$
\sum_{\rho} L_{\tau}(\lambda, \rho)=\frac{1}{\operatorname{dim}(\lambda) \operatorname{dim}(\tau)|G|} \sum_{g} \chi^{\lambda}(g) \chi^{\tau}(g) \sum_{\rho} \chi^{\rho}(i d) \overline{\chi^{\rho}(g)}=1 .
$$

For the reversibility assertion, the fact that $\chi^{\tau}$ and the transition probabilities of $L_{\tau}$ are both real valued implies that

$$
\frac{\operatorname{dim}(\lambda)^{2}}{|G|} L_{\tau}(\lambda, \rho)=\frac{\operatorname{dim}(\rho)^{2}}{|G|} L_{\tau}(\rho, \lambda) .
$$


An exchangeable pair $\left(W, W^{\prime}\right)$ is now constructed from the chain $L_{\tau}$ in the standard way. First choose $\lambda$ from the Plancherel measure of $G$, then choose $\rho$ with probability $L_{\tau}(\lambda, \rho)$, and finally let $\left(W, W^{\prime}\right)=(W(\lambda), W(\rho))$. Note that if $\tau$ is the trivial representation, then $W^{\prime}=W$. Since Stein's method is in the spirit of Taylor approximation, it is good for $W^{\prime}$ to be close to $W$, so in examples $\tau$ should typically be chosen to be as close as possible to the trivial representation.

The remaining results in this subsection show that the exchangeable pair $\left(W, W^{\prime}\right)$ has desirable properties.

Lemma 3.7. $\mathbb{E}\left(W^{\prime} \mid W\right)=\left(\frac{\chi^{\tau}(C)}{\operatorname{dim}(\tau)}\right) W$.

Proof. From the definition of $W^{\prime}$,

$$
\begin{aligned}
\mathbb{E}\left(W^{\prime} \mid \lambda\right) & =|C|^{1 / 2} \sum_{\rho} \frac{\operatorname{dim}(\rho)}{\operatorname{dim}(\lambda) \operatorname{dim}(\tau)} \frac{1}{|G|} \sum_{g} \chi^{\lambda}(g) \chi^{\tau}(g) \overline{\chi^{\rho}(g)} \frac{\chi^{\rho}(C)}{\operatorname{dim}(\rho)} \\
& =\frac{|C|^{1 / 2}}{|G|} \sum_{g} \frac{\chi^{\lambda}(g)}{\operatorname{dim}(\lambda)} \frac{\chi^{\tau}(g)}{\operatorname{dim}(\tau)} \sum_{\rho} \chi^{\rho}(C) \overline{\chi^{\rho}(g)} \\
& =\left(\frac{\chi^{\tau}(C)}{\operatorname{dim}(\tau)}\right) W(\lambda) .
\end{aligned}
$$

The second equality switched the order of summation and the last step is Lemma 3.3. The result follows since this depends on $\lambda$ only through $W$.

Corollary 3.8 is not needed in what follows, but is worth recording.

Corollary 3.8. The eigenvalues of $L_{\tau}$ are $\frac{\chi^{\tau}(C)}{\operatorname{dim}(\tau)}$ as $C$ ranges over conjugacy classes of $G$. The functions $\psi_{C}(\lambda)=\frac{|C|^{1 / 2} \chi^{\lambda}(C)}{\operatorname{dim}(\lambda)}$ are a basis of eigenvectors of $L_{\tau}$, orthonormal with respect to the inner product

$$
\left\langle f_{1}, f_{2}\right\rangle=\sum_{\lambda} f_{1}(\lambda) \overline{f_{2}(\lambda)} \frac{\operatorname{dim}(\lambda)^{2}}{|G|} .
$$

Proof. The proof of Lemma 3.7 shows that $\psi_{C}$ is an eigenvector of $L_{\tau}$ with eigenvalue $\frac{\chi^{\tau}(C)}{\operatorname{dim}(\tau)}$. The orthonormality assertion follows from Lemma 3.3 and the basis assertion follows since the number of conjugacy classes of $G$ is equal to the number of irreducible representations of $G$.

Lemma 3.9. $\mathbb{E}\left(W^{\prime}-W\right)^{2}=2\left(1-\frac{\chi^{\tau}(C)}{\operatorname{dim}(\tau)}\right)$.

Proof. This is immediate from Lemmas 2.3 and 3.7 .

For the remainder of this subsection, if $K$ is a conjugacy class of $G, p_{m}(K)$ will denote the probability that the random walk generated by $C$ started at the identity is in $K$ after $m$ steps.

We remark that part (2) of Lemma 3.10 writes $\operatorname{Var}\left(\mathbb{E}\left[\left(W^{\prime}-W\right)^{2} \mid \lambda\right]\right)$ as a sum of positive quantities.

\section{Lemma 3.10.}

(1)

$$
\mathbb{E}\left[\left(W^{\prime}-W\right)^{2} \mid \lambda\right]=|C| \sum_{K} p_{2}(K)\left(\frac{\chi^{\tau}(K)}{\operatorname{dim}(\tau)}+1-\frac{2 \chi^{\tau}(C)}{\operatorname{dim}(\tau)}\right) \frac{\chi^{\lambda}(K)}{\operatorname{dim}(\lambda)}
$$

where the sum is over all conjugacy classes $K$ of $G$. 
$(2)$

$$
\operatorname{Var}\left(\mathbb{E}\left[\left(W^{\prime}-W\right)^{2} \mid \lambda\right]\right)=|C|^{2} \sum_{K \neq i d} \frac{p_{2}(K)^{2}}{|K|}\left(\frac{\chi^{\tau}(K)}{\operatorname{dim}(\tau)}+1-\frac{2 \chi^{\tau}(C)}{\operatorname{dim}(\tau)}\right)^{2}
$$

where $K$ ranges over all non-identity conjugacy classes of $G$.

Proof. Observe that

$$
\begin{aligned}
\mathbb{E}\left(\left(W^{\prime}\right)^{2} \mid \lambda\right) & =\frac{1}{|G|} \sum_{\rho} \operatorname{dim}(\rho) \sum_{g} \frac{\chi^{\lambda}(g)}{\operatorname{dim}(\lambda)} \frac{\chi^{\tau}(g)}{\operatorname{dim}(\tau)} \overline{\chi^{\rho}(g)}\left(\frac{|C|^{1 / 2} \chi^{\rho}(C)}{\operatorname{dim}(\rho)}\right)^{2} \\
& =|C| \sum_{g} \frac{\chi^{\lambda}(g)}{\operatorname{dim}(\lambda)} \frac{\chi^{\tau}(g)}{\operatorname{dim}(\tau)} \cdot \frac{1}{|G|} \sum_{\rho} \operatorname{dim}(\rho) \overline{\chi^{\rho}(g)}\left(\frac{\chi^{\rho}(C)}{\operatorname{dim}(\rho)}\right)^{2} \\
& =|C| \sum_{K} p_{2}(K) \frac{\chi^{\tau}(K)}{\operatorname{dim}(\tau)} \frac{\chi^{\lambda}(K)}{\operatorname{dim}(\lambda)} .
\end{aligned}
$$

The second equality switched the order of summation, and the final equality was Lemma 3.4

Next we claim that $W^{2}=|C| \sum_{K} p_{2}(K) \frac{\chi^{\lambda}(K)}{\operatorname{dim}(\lambda)}$. To see this note from Lemma 3.4 that

$$
\frac{|K|}{|G|} \sum_{\rho} \frac{\chi^{\rho}(C)^{2} \overline{\chi^{\rho}(K)}}{\operatorname{dim}(\rho)}=p_{2}(K) .
$$

Multiplying both sides by $\frac{|C| \chi^{\lambda}(K)}{\operatorname{dim}(\lambda)}$ and summing over all $K$, the claimed expression for $W^{2}$ follows from Lemma 3.2

Now note from Lemma 3.7 that

$$
\begin{aligned}
\mathbb{E}\left[\left(W^{\prime}-W\right)^{2} \mid \lambda\right] & =\mathbb{E}\left(\left(W^{\prime}\right)^{2} \mid \lambda\right)-2 W \mathbb{E}\left(W^{\prime} \mid \lambda\right)+W^{2} \\
& =\mathbb{E}\left(\left(W^{\prime}\right)^{2} \mid \lambda\right)+\left(1-\frac{2 \chi^{\tau}(C)}{\operatorname{dim}(\tau)}\right) W^{2} .
\end{aligned}
$$

The first part of the lemma follows from this equation and the first two paragraphs.

From the first part of the lemma and Lemma 3.3 , it follows that

$$
\mathbb{E}\left(\mathbb{E}\left[\left(W^{\prime}-W\right)^{2} \mid \lambda\right]^{2}\right)=|C|^{2} \sum_{K} \frac{p_{2}(K)^{2}}{|K|}\left(\frac{\chi^{\tau}(K)}{\operatorname{dim}(\tau)}+1-\frac{2 \chi^{\tau}(C)}{\operatorname{dim}(\tau)}\right)^{2} .
$$

Note that if $K$ is the identity class, then $p_{2}(K)=\frac{1}{|C|}$, so that the contribution coming from the identity class is $4\left(1-\frac{\chi^{\tau}(C)}{\operatorname{dim}(\tau)}\right)^{2}$. The second part of the lemma follows since by Lemma 3.9 .

$$
\operatorname{Var}\left(\mathbb{E}\left[\left(W^{\prime}-W\right)^{2} \mid \lambda\right]\right)=\mathbb{E}\left(\mathbb{E}\left[\left(W^{\prime}-W\right)^{2} \mid \lambda\right]^{2}\right)-4\left(1-\frac{\chi^{\tau}(C)}{\operatorname{dim}(\tau)}\right)^{2}
$$

Lemma 3.11. Let $k$ be a positive integer.

(1) $\mathbb{E}\left(W^{\prime}-W\right)^{k}=|C|^{k / 2} \sum_{m=0}^{k}(-1)^{k-m}\left(\begin{array}{l}k \\ m\end{array}\right) \sum_{K} \frac{\chi^{\tau}(K)}{\operatorname{dim}(\tau)} \frac{p_{m}(K) p_{k-m}(K)}{|K|}$.

(2) $\mathbb{E}\left(W^{\prime}-W\right)^{4}$ is equal to

$$
|C|^{2} \sum_{K}\left[8\left(1-\frac{\chi^{\tau}(C)}{\operatorname{dim}(\tau)}\right)-6\left(1-\frac{\chi^{\tau}(K)}{\operatorname{dim}(\tau)}\right)\right] \frac{p_{2}(K)^{2}}{|K|} .
$$


Proof. For the first assertion, note that

$$
\begin{aligned}
\mathbb{E}\left(\left(W^{\prime}-W\right)^{k} \mid \lambda\right)= & \frac{|C|^{k / 2}}{\operatorname{dim}(\lambda) \operatorname{dim}(\tau)} \sum_{\rho} \frac{\operatorname{dim}(\rho)}{|G|} \sum_{g} \chi^{\lambda}(g) \chi^{\tau}(g) \overline{\chi^{\rho}(g)} \\
& \cdot \sum_{m=0}^{k}(-1)^{k-m}\left(\begin{array}{c}
k \\
m
\end{array}\right)\left(\frac{\chi^{\rho}(C)}{\operatorname{dim}(\rho)}\right)^{m}\left(\frac{\chi^{\lambda}(C)}{\operatorname{dim}(\lambda)}\right)^{k-m} \\
= & \frac{|C|^{k / 2}}{\operatorname{dim}(\lambda) \operatorname{dim}(\tau)} \sum_{m=0}^{k}(-1)^{k-m}\left(\begin{array}{c}
k \\
m
\end{array}\right)\left(\frac{\chi^{\lambda}(C)}{\operatorname{dim}(\lambda)}\right)^{k-m} \\
& \cdot \sum_{g} \chi^{\tau}(g) \chi^{\lambda}(g) \sum_{\rho} \frac{\operatorname{dim}(\rho)}{|G|}\left(\frac{\chi^{\rho}(C)}{\operatorname{dim}(\rho)}\right)^{m} \overline{\chi^{\rho}(g)} \\
= & \frac{|C|^{k / 2}}{\operatorname{dim}(\lambda) \operatorname{dim}(\tau)} \sum_{m=0}^{k}(-1)^{k-m}\left(\begin{array}{c}
k \\
m
\end{array}\right)\left(\frac{\chi^{\lambda}(C)}{\operatorname{dim}(\lambda)}\right)^{k-m} \\
& \cdot \sum_{K} \chi^{\tau}(K) \chi^{\lambda}(K) p_{m}(K) .
\end{aligned}
$$

The second step switched the order of summation and the final equality is by Lemma 3.4. Thus $\mathbb{E}\left(\left(W^{\prime}-W\right)^{k}\right)$ is equal to

$$
\begin{aligned}
\mathbb{E}\left(\mathbb{E}\left(\left(W^{\prime}-W\right)^{k} \mid \lambda\right)\right)= & |C|^{k / 2} \sum_{m=0}^{k}(-1)^{k-m}\left(\begin{array}{c}
k \\
m
\end{array}\right) \sum_{K} p_{m}(K) \frac{\chi^{\tau}(K)}{\operatorname{dim}(\tau)} \\
& \cdot \sum_{\lambda} \frac{\operatorname{dim}(\lambda)^{2}}{|G|} \frac{\chi^{\lambda}(K)}{\operatorname{dim}(\lambda)}\left(\frac{\chi^{\lambda}(C)}{\operatorname{dim}(\lambda)}\right)^{k-m} .
\end{aligned}
$$

The first assertion now follows from Lemma 3.4 and the fact that $\chi^{\lambda}(C)$ is real for all $\lambda$.

For the second assertion, note by the first assertion that

$$
\mathbb{E}\left(W^{\prime}-W\right)^{4}=|C|^{2} \sum_{m=0}^{4}(-1)^{m}\left(\begin{array}{c}
4 \\
m
\end{array}\right) \sum_{K} \frac{\chi^{\tau}(K)}{\operatorname{dim}(\tau)} \frac{p_{m}(K) p_{4-m}(K)}{|K|} .
$$

If $\tau$ is the trivial representation, then $W^{\prime}=W$, which implies that

$$
0=|C|^{2} \sum_{m=0}^{4}(-1)^{m}\left(\begin{array}{c}
4 \\
m
\end{array}\right) \sum_{K} \frac{p_{m}(K) p_{4-m}(K)}{|K|} .
$$

Thus for general $\tau$,

$$
\mathbb{E}\left(W^{\prime}-W\right)^{4}=-|C|^{2} \sum_{m=0}^{4}(-1)^{m}\left(\begin{array}{c}
4 \\
m
\end{array}\right) \sum_{K}\left(1-\frac{\chi^{\tau}(K)}{\operatorname{dim}(\tau)}\right) \frac{p_{m}(K) p_{4-m}(K)}{|K|} .
$$

Observe that the $m=0,4$ terms in this sum vanish, since the only contribution could come from the identity, which contributes 0 . The $m=2$ term is

$$
-6|C|^{2} \sum_{K}\left(1-\frac{\chi^{\tau}(K)}{\operatorname{dim}(\tau)}\right) \frac{p_{2}(K)^{2}}{|K|} .
$$


The $m=1,3$ terms are equal and together contribute

$$
\begin{aligned}
& 8|C|^{2} \sum_{K}\left(1-\frac{\chi^{\tau}(K)}{\operatorname{dim}(\tau)}\right) \frac{p_{1}(K) p_{3}(K)}{|K|} \\
= & 8|C|\left(1-\frac{\chi^{\tau}(C)}{\operatorname{dim}(\tau)}\right) p_{3}(C) \\
= & 8|C|^{2}\left(1-\frac{\chi^{\tau}(C)}{\operatorname{dim}(\tau)}\right) p_{4}(i d) \\
= & 8|C|^{2}\left(1-\frac{\chi^{\tau}(C)}{\operatorname{dim}(\tau)}\right) \sum_{K} \frac{p_{2}(K)^{2}}{|K|},
\end{aligned}
$$

where $i d$ is the identity and the last two equalities can be seen either directly or from Lemma 3.4. This completes the proof of the second assertion.

Putting the pieces together, one obtains the following theorem.

Theorem 3.12. Let $C$ be a conjugacy class of a finite group $G$ such that $C=C^{-1}$ and fix an irreducible representation $\tau$ of $G$ whose character is real valued, and such that $0<\frac{\chi^{\tau}(C)}{\operatorname{dim}(\tau)}<1$. Let $\lambda$ be a random irreducible representation, chosen from the Plancherel measure of $G$. Let $W=\frac{|C|^{1 / 2} \chi^{\lambda}(C)}{\operatorname{dim}(\lambda)}$. Then for all real $x_{0}$,

$$
\begin{aligned}
& \left|\mathbb{P}\left(W \leq x_{0}\right)-\frac{1}{\sqrt{2 \pi}} \int_{-\infty}^{x_{0}} e^{-\frac{x^{2}}{2}} d x\right| \\
\leq & \frac{|C|}{a} \sqrt{\sum_{K \neq i d} \frac{p_{2}(K)^{2}}{|K|}\left(\frac{\chi^{\tau}(K)}{\operatorname{dim}(\tau)}+2 a-1\right)^{2}} \\
& +\left[\frac{|C|^{2}}{\pi} \sum_{K}\left(8-\frac{6}{a}\left(1-\frac{\chi^{\tau}(K)}{\operatorname{dim}(\tau)}\right)\right) \frac{p_{2}(K)^{2}}{|K|}\right]^{1 / 4},
\end{aligned}
$$

where $a=1-\frac{\chi^{\tau}(C)}{\operatorname{dim}(\tau)}$.

Proof. One applies Theorem 2.1 to the exchangeable pair $\left(W, W^{\prime}\right)$ of this subsection. To handle the first term in Theorem 2.1. note that the conditional version of Jensen's inequality (Section 4.1 of [11]) implies that

$$
\operatorname{Var}\left(\mathbb{E}\left[\left(W^{\prime}-W\right)^{2} \mid W\right]\right) \leq \operatorname{Var}\left(\mathbb{E}\left[\left(W^{\prime}-W\right)^{2} \mid \lambda\right]\right) .
$$

Part (2) of Lemma 3.10 then gives the first term in the theorem. To upper bound the second term in Theorem 2.1 note by the Cauchy-Schwarz inequality that

$$
\mathbb{E}\left|W^{\prime}-W\right|^{3} \leq \sqrt{\mathbb{E}\left(W^{\prime}-W\right)^{2} \mathbb{E}\left(W^{\prime}-W\right)^{4}} .
$$

Now use Lemma 3.9 and part (2) of Lemma 3.11 .

3.3. Example: Cayley graphs of the symmetric group. This subsection applies the theory of Subsection 3.2 to the case where the group is $S_{n}$ and $C$ is the conjugacy class of $i$-cycles.

It is useful to recall some facts about the symmetric group. Since $K=K^{-1}$ for all conjugacy classes $K$ of $S_{n}$, Lemma 3.1 implies that all irreducible characters of $S_{n}$ are real valued. Also it is elementary that $|K|=\frac{n !}{\prod_{j} j^{m_{j}} m_{j} !}$, where $m_{j}$ is the number of cycles of length $j$ of an element of $K$. 
In order to upper bound the error terms in Theorem 3.12, the following estimate on the two step transition probabilities of the random walk generated by $C$ will be useful.

Lemma 3.13. Let $C$ be the conjugacy class of cycles of length $i$ of the symmetric group $S_{n}$. Then for $i$ fixed and $n \geq 2 i, \frac{p_{2}(K)^{2}}{|K|}$ is equal to

(1) $i^{2} n^{-2 i}+O\left(n^{-2 i-1}\right)$ if $K$ is the identity conjugacy class.

(2) $2 i^{2} n^{-2 i}+O\left(n^{-2 i-1}\right)$ if $K$ is the conjugacy class consisting of two cycles of length $i$.

(3) $O\left(n^{-2 i-1}\right)$ otherwise.

Proof. The first assertion is clear since if $K$ is the identity class, $p_{2}(K)=\frac{1}{|C|}$. For the second assertion, note that $p_{2}(K)=1+O\left(n^{-1}\right)$ since the only way that a product of two $i$-cycles is not in $K$ is if the $i$-cycles have a symbol in common.

For the third assertion, there are two cases. The first case is that $K$ has exactly $n-2 i$ fixed points. Then $|K|$ is at least $c_{i} n^{2 i}$ where $c_{i}$ is a constant depending on $i$. Since $K$ is not the class consisting of exactly two $i$-cycles, $p_{2}(K)=O\left(n^{-1}\right)$, proving the third assertion in this case. The second case is that $K$ has $n-2 i+r$ fixed points, where $1 \leq r<2 i$. Then $|K|$ is at least $c_{i} n^{2 i-r}$ where $c_{i}$ is a constant depending on $i$. However $p_{2}(K)=O\left(n^{-r}\right)$, since in the product of the two $i$-cycles, there are $r$ symbols moved by the first $i$-cycle each of which is mapped back to itself by the second $i$-cycle. Thus in this case also, the third assertion is proved.

Theorem 3.14. Let $C$ be the conjugacy class of $i$-cycles in $S_{n}$, with $i \geq 2$. Choosing $\lambda$ from the Plancherel measure of the symmetric group, define a random variable $W=\frac{\sqrt{\left(\begin{array}{c}n \\ i\end{array}\right)(i-1) !} \chi^{\lambda}(C)}{\operatorname{dim}(\lambda)}$. Then there is a constant $A_{i}$ such that for all real $x_{0}$,

$$
\left|\mathbb{P}\left(W \leq x_{0}\right)-\frac{1}{\sqrt{2 \pi}} \int_{-\infty}^{x_{0}} e^{-\frac{x^{2}}{2}} d x\right| \leq A_{i} n^{-1 / 4} .
$$

Proof. One applies Theorem 3.12, choosing $\tau$ to be the irreducible representation corresponding to $\tau=(n-1,1)$. Then $\chi^{\tau}(K)$ is the number of fixed points of $K$ $-1, \operatorname{dim}(\tau)=n-1$, and $a=1-\frac{\chi^{\tau}(C)}{\operatorname{dim}(\tau)}=\frac{i}{n-1}$. Note that for any non-identity conjugacy class $K$,

$$
\frac{p_{2}(K)^{2}}{|K|}\left(\frac{\chi^{\tau}(K)}{\operatorname{dim}(\tau)}+2 a-1\right)^{2}=O\left(n^{-2 i-3}\right) .
$$

This follows from Lemma 3.13 and the fact that $\frac{\chi^{\tau}(K)}{\operatorname{dim}(\tau)}+2 a-1$ vanishes if $K$ has $n-2 i$ fixed points and is $O\left(n^{-1}\right)$ for any other class $K$ with $p_{2}(K) \neq 0$, since such a class has at least $n-2 i$ fixed points. Since $|C|=\frac{n !}{(n-i) ! i}$ and $a=\frac{i}{n-1}$, it follows that the first error term in Theorem 3.12 is at most $A_{i}^{\prime} n^{-1 / 2}$, where $A_{i}^{\prime}$ is a constant depending on $i$.

To bound the second error term in Theorem 3.12, observe that Lemma 3.13 implies that

$$
\sum_{K}\left(8-\frac{6}{a}\left(1-\frac{\chi^{\tau}(K)}{\operatorname{dim}(\tau)}\right)\right) \frac{p_{2}(K)^{2}}{|K|}
$$

is $O\left(n^{-2 i-1}\right)$. Indeed, the identity class contributes $8 i^{2} n^{-2 i}+O\left(n^{-2 i-1}\right)$ and the class of two $i$-cycles contributes $-8 i^{2} n^{-2 i}+O\left(n^{-2 i-1}\right)$. All other $K$ for which 
$p_{2}(K) \neq 0$ have at least $n-2 i$ fixed points, so $\left(8-\frac{6}{a}\left(1-\frac{\chi^{\tau}(K)}{\operatorname{dim}(\tau)}\right)\right)$ is $O(1)$ and it follows from Lemma 3.13 that the contribution of any such $K$ is $O\left(n^{-2 i-1}\right)$. The same is true for the combined contribution of all such $K$ since the number of $K$ with at least $n-2 i$ fixed points is bounded by a constant depending on $i$. Hence the second error term in Theorem 3.12 is at most $A_{i}^{\prime \prime} n^{-1 / 4}$ where $A_{i}^{\prime \prime}$ is a constant depending only on $i$.

To conclude this example, we note that there are many conjugacy classes $C$ of the symmetric group for which $W$ does not have a normal limit (see 21] for a detailed discussion of the limit laws). In fact even if $C$ has $n-i$ fixed points (like the class of $i$-cycles), it need not be the case that the distribution of $W$ is approximately normal.

\section{Gelfand PAIRS}

If $G$ is a finite group and $K$ is a subgroup of $G$ such that the induced representation $1_{K}^{G}$ is multiplicity free, then the pair $(G, K)$ is called a Gelfand pair. Gelfand pairs have applications in number theory (see [18, for a survey). Chapter 3 of $[8]$ shows that Gelfand pairs are useful for studying the convergence rate of random walks on $G / K$. The purpose of this section is to show that the results of Section 3 extend to the setting of Gelfand pairs, and can be used to study the spectrum of random walks on $G / K$.

Subsection 4.1 discusses the representation theory of Gelfand pairs. Subsection 4.2 derives a general central limit theorem for random spherical functions of a Gelfand pair. Subsection 4.3 illustrates the theory on a toy example, proving a central limit theorem for the spectrum of the hypercube. A more serious example is considered in Subsection 4.4, which obtains a new central limit theorem for the spectrum of certain random walks on the perfect matchings of $2 \mathrm{n}$ symbols. As indicated there, this result can be stated in terms of Jack ${ }_{2}$ measure, which is of interest to workers in random matrix theory.

4.1. Background from representation theory. We discuss some facts about the representation theory of Gelfand pairs. A useful reference is Chapter 7 of [29].

The induced representation $1_{K}^{G}$ decomposes in a multiplicity free way as $\bigoplus_{r=0}^{s} V_{r}$, where $V_{0}$ denotes the trivial representation of $G$. Let $d_{r}$ denote the dimension of $V_{r}$. For $0 \leq r \leq s$, let $\omega_{r}$ denote the corresponding spherical function on $G$, defined by

$$
\omega_{r}(x)=\frac{1}{|K|} \sum_{k \in K} \chi^{(r)}\left(x^{-1} k\right)
$$

where $\chi^{(r)}$ is the character of $V_{r}$. The functions $\omega_{r}$ are a basis of the space of functions on $G$ which are constant on the double cosets of $K$ in $G$. Let $K_{0}, \cdots, K_{s}$ denote the double cosets of $K$ in $G$ and let $g_{0}, \cdots, g_{s}$ be corresponding double coset representatives, so that $K_{i}=K g_{i} K$. It is convenient to take $g_{0}$ to be the identity element of $G$. From page 389 of [29], one has that $\omega_{i}\left(g_{0}\right)=1$ for all $i$.

Lemma 4.1 (29], page 389). Let $\omega$ be a spherical function of the Gelfand pair $(G, K)$. Then $\omega\left(x^{-1}\right)=\overline{\omega(x)}$.

The following two orthogonality relations are also useful. 
Lemma 4.2 (29], page 389). For $0 \leq i, j \leq s$,

$$
\frac{d_{i}}{|G|} \sum_{r=0}^{s}\left|K_{r}\right| \omega_{i}\left(g_{r}\right) \overline{\omega_{j}\left(g_{r}\right)}=\delta_{i, j}
$$

Lemma 4.3. For $0 \leq r, t \leq s$,

$$
\sum_{i=0}^{s} d_{i} \omega_{i}\left(g_{r}\right) \overline{\omega_{i}\left(g_{t}\right)}=\delta_{r, t} \frac{|G|}{\left|K_{r}\right|}
$$

Proof. Consider the $s \times s$ matrix whose entry in the $i$ th column and $r$ th row is $\sqrt{\frac{d_{i}\left|K_{r}\right|}{|G|}} \omega_{i}\left(g_{r}\right)$. By Lemma 4.2, the columns of this matrix are orthonormal. Hence so are its rows, proving the lemma.

If $P$ is a $K$ biinvariant probability on $G$ (i.e. constant on double cosets of $K$ in $G)$, let $p_{m}\left(K_{r}\right)$ denote the probability that the $m$-fold convolution of $P$ assigns to the double coset $K g_{r} K$. Lemma 4.4 is an analog of Lemma 3.4 and could be proved along similar lines, as in [19. Instead, we use the language of Fourier analysis, as developed on page 395 of 29 .

Lemma 4.4. Let $P$ be the $K$ biinvariant probability on $G$ which associates mass 1 to the double coset $K_{u}$ and mass 0 to all other $K$ double cosets of $G$. Then for $0 \leq r \leq s$,

$$
p_{m}\left(K_{r}\right)=\left|K_{r}\right| \sum_{i=0}^{s} \frac{d_{i}}{|G|} \omega_{i}\left(g_{u}\right)^{m} \overline{\omega_{i}\left(g_{r}\right)} .
$$

Proof. If $f$ is a complex valued $K$ biinvariant function on $G$, define $\hat{f}\left(\omega_{i}\right)$ (the Fourier transform of $f$ at the spherical function $\omega_{i}$ ) by

$$
\hat{f}\left(\omega_{i}\right)=\sum_{g \in G} f(g) \overline{\omega_{i}(g)} .
$$

Then the Fourier inversion theorem gives that

$$
f=\frac{1}{|G|} \sum_{i=0}^{s} \hat{f}\left(\omega_{i}\right) d_{i} \omega_{i} .
$$

It is also true that the Fourier transform of the convolution of two $\mathrm{K}$ biinvariant functions is the product of their Fourier transforms. Thus the $m$-fold convolution of $f$ is equal to

$$
\frac{1}{|G|} \sum_{i=0}^{s}\left(\hat{f}\left(\omega_{i}\right)\right)^{m} d_{i} \omega_{i} .
$$

The lemma now follows by taking $f=P$.

To connect the study of random spherical functions with spectral graph theory, suppose for convenience that $(G, K)$ is a symmetric Gelfand pair, which means that $K g K=K g^{-1} K$ for all $g$. This condition holds for the examples in Subsections 4.3 and 4.4. Then fixing a double coset $K_{u}$, one can define a graph $H_{u}$ whose vertices are the right cosets of $K$ by connecting $K h_{1}$ to $K h_{2}$ if and only if $K h_{1} h_{2}^{-1} K=K_{u}$. A more general construction appears in 28]. The graph $H_{u}$ is vertex transitive, since $G$ acts transitively on the right cosets of $K$ by sending $K h$ to $K h g^{-1}$, and $K h_{1}$ is connected to $K h_{2}$ if and only if $K h_{1} g^{-1}$ is connected to $K h_{2} g^{-1}$. Lemma 4.5 determines the spectrum of random walk on $H_{u}$. 
Lemma 4.5. Let $(G, K)$ be a symmetric Gelfand pair. Then for any double coset $K_{u}$, the random walk on the graph $H_{u}$ has eigenvalues $\omega_{i}\left(g_{u}\right)$ occurring with multiplicity $d_{i}$ for $0 \leq i \leq s$.

Proof. Let $M$ be the transition matrix for the random walk on $H_{u}$. Since $H_{u}$ is vertex transitive, the trace of $M^{k}$ is $|G| /|K|$ multiplied by the probability that the random walk on $H_{u}$ started at the right coset $K$ is at $K$ after $k$ steps. By Theorem 7.5 of 28 , this return probability is

$$
\frac{|K|}{|G|} \sum_{i=0}^{s} d_{i} \omega_{i}\left(g_{u}\right)^{k}
$$

so that the trace of $M^{k}$ is $\sum_{i=0}^{s} d_{i} \omega_{i}\left(g_{u}\right)^{k}$. Since this is true for all $k \geq 0$, the result follows.

Note that since $d_{0}+\cdots+d_{s}=|G / K|$, one can define a probability measure on $\{0, \cdots, s\}$ (or equivalently on the set $\left\{\omega_{0}, \cdots, \omega_{s}\right\}$ ) by choosing $i$ with probability $\frac{d_{i}|K|}{|G|}$. This is the Gelfand pair analog of the Plancherel measure of a finite group, and in this paper it will be referred to as Plancherel measure. Lemma 4.5 showed that if $(G, K)$ is a symmetric Gelfand pair, then the eigenvalues of certain graphs on $G / K$ occur with multiplicity proportional to Plancherel measure.

4.2. Central limit theorem for spherical functions. The aim of this subsection is to prove a central limit theorem for the random variable $W$ defined by $W(i)=$ $\frac{\left|K_{u}\right|^{1 / 2}}{|K|^{1 / 2}} \omega_{i}\left(g_{u}\right)$. Here $g_{u}$ is fixed satisfying $K g_{u} K=K g_{u}^{-1} K$ and $i$ is random from the Plancherel measure of the Gelfand pair $(G, K)$. Lemma 4.1 implies that $W$ is real valued. It is not assumed that the Gelfand pair $(G, K)$ is symmetric. Note that by Lemma 4.3 $\mathbb{E}(W)=0$ if $K g_{u} K \neq K$, and $\mathbb{E}\left(W^{2}\right)=1$.

To construct an exchangeable pair to be used for Stein's method, it is helpful to define a Markov chain on the set of spherical functions of $(G, K)$. Motivated by the construction in Subsection 3.2. we proceed as follows. Fix $t$ with $0 \leq t \leq s$ such that the spherical function $\omega_{t}$ is real-valued. Let $L_{t}$ be the Markov chain on the set $\left\{\omega_{0}, \cdots, \omega_{s}\right\}$ which transitions from $\omega_{i}$ to $\omega_{j}$ with probability

$$
L_{t}\left(\omega_{i}, \omega_{j}\right):=\frac{d_{j}}{|G|} \sum_{r=0}^{s}\left|K_{r}\right| \omega_{i}\left(g_{r}\right) \omega_{t}\left(g_{r}\right) \overline{\omega_{j}\left(g_{r}\right)} .
$$

Lemma 4.6 verifies that $L_{t}$ is a Markov chain which is reversible with respect to Plancherel measure.

Lemma 4.6. Let $t$ be such that the spherical function $\omega_{t}$ is real-valued. Then the transition probabilities of $L_{t}$ are real and non-negative and sum to 1 . Moreover the chain $L_{t}$ is reversible with respect to the Plancherel measure of the pair $(G, K)$.

Proof. From page 396 of [29], if $a_{i t}^{k}$ are defined by

$$
\omega_{i} \omega_{t}=\sum_{k=0}^{s} a_{i t}^{k} \omega_{k}
$$

(where the notation $\omega_{i} \omega_{t}$ denotes the pointwise product), then $a_{i t}^{k}$ are real and non-negative. Hence Lemma 4.2 implies that $L_{t}\left(\omega_{i}, \omega_{j}\right)$ is real and non-negative. 
Since $\omega_{j}\left(g_{0}\right)=1$ for all $j$, Lemma 4.3 gives that

$$
\sum_{j=0}^{s} L_{t}\left(\omega_{i}, \omega_{j}\right)=\sum_{r=0}^{s}\left|K_{r}\right| \omega_{i}\left(g_{r}\right) \omega_{t}\left(g_{r}\right) \frac{1}{|G|} \sum_{j=0}^{s} d_{j} \omega_{j}\left(g_{0}\right) \overline{\omega_{j}\left(g_{r}\right)}=1 .
$$

Reversibility of $L_{t}$ with respect to Plancherel measure is equivalent to showing that

$$
\frac{d_{i} d_{j}|K|}{|G|} \sum_{r=0}^{s}\left|K_{r}\right| \omega_{i}\left(g_{r}\right) \omega_{t}\left(g_{r}\right) \overline{\omega_{j}\left(g_{r}\right)}=\frac{d_{i} d_{j}|K|}{|G|} \sum_{r=0}^{s}\left|K_{r}\right| \omega_{j}\left(g_{r}\right) \omega_{t}\left(g_{r}\right) \overline{\omega_{i}\left(g_{r}\right)} .
$$

Both sides are real by the previous paragraph, so the result follows by the assumption that $\omega_{t}$ is real valued.

An exchangeable pair $\left(W, W^{\prime}\right)$ to be used in a Stein's method approach to studying $W$ can be constructed from the chain $L_{t}$ in the usual way. First choose $i$ from Plancherel measure, then choose $j$ with probability $L_{t}\left(\omega_{i}, \omega_{j}\right)$, and finally let $\left(W, W^{\prime}\right)=\left(W\left(\omega_{i}\right), W\left(\omega_{j}\right)\right)$. As in the group case, it is useful to have $W^{\prime}$ close to $W$, and thus one typically wants to choose $\omega_{t}$ as close as possible to the trivial spherical function.

Lemma 4.7. $\mathbb{E}\left(W^{\prime} \mid W\right)=\omega_{t}\left(g_{u}\right) W$.

Proof. From the definitions and Lemma 4.3 .

$$
\begin{aligned}
\mathbb{E}\left(W^{\prime} \mid \omega_{i}\right) & =\frac{\left|K_{u}\right|^{1 / 2}}{|K|^{1 / 2}} \sum_{j=0}^{s} \frac{d_{j}}{|G|} \sum_{r=0}^{s}\left|K_{r}\right| \omega_{i}\left(g_{r}\right) \omega_{t}\left(g_{r}\right) \overline{\omega_{j}\left(g_{r}\right)} \omega_{j}\left(g_{u}\right) \\
& =\frac{\left|K_{u}\right|^{1 / 2}}{|K|^{1 / 2}} \sum_{r=0}^{s}\left|K_{r}\right| \omega_{i}\left(g_{r}\right) \omega_{t}\left(g_{r}\right) \sum_{j=0}^{s} \frac{d_{j}}{|G|} \omega_{j}\left(g_{u}\right) \overline{\omega_{j}\left(g_{r}\right)} \\
& =\omega_{t}\left(g_{u}\right) W\left(\omega_{i}\right) .
\end{aligned}
$$

The result follows since this depends on $\omega_{i}$ only through $W$.

Corollary 4.8 is not needed in the sequel but is interesting.

Corollary 4.8. The eigenvalues of $L_{t}$ are $\omega_{t}\left(g_{r}\right)$ for $0 \leq r \leq s$. The functions $\psi_{r}\left(\omega_{i}\right)=\frac{\left|K_{g_{r}}\right|^{1 / 2}}{|K|^{1 / 2}} \omega_{i}\left(g_{r}\right)$ are a basis of eigenvectors of $L_{\tau}$, orthonormal with respect to the inner product

$$
\left\langle f_{1}, f_{2}\right\rangle=\sum_{i=0}^{s} f_{1}\left(\omega_{i}\right) \overline{f_{2}\left(\omega_{i}\right)} \frac{d_{i}|K|}{|G|} .
$$

Proof. By the proof of Lemma 4.7, $\psi_{r}$ is an eigenvector of $L_{t}$ with eigenvalue $\omega_{t}\left(g_{r}\right)$. The orthonormality assertion follows from Lemma 4.3. and the basis assertion follows since the number of spherical functions is equal to the number of $K$ double cosets of $G$.

Lemma 4.9. $\mathbb{E}\left(W^{\prime}-W\right)^{2}=2\left(1-\omega_{t}\left(g_{u}\right)\right)$.

Proof. This is immediate from Lemmas 2.3 and 4.7.

Lemma 4.10.

$$
\mathbb{E}\left[\left(W^{\prime}-W\right)^{2} \mid \omega_{i}\right]=\frac{\left|K_{u}\right|}{|K|} \sum_{r=0}^{s} p_{2}\left(K_{r}\right)\left(\omega_{t}\left(g_{r}\right)+1-2 \omega_{t}\left(g_{u}\right)\right) \omega_{i}\left(g_{r}\right) .
$$


$(2)$

$$
\operatorname{Var}\left(\mathbb{E}\left[\left(W^{\prime}-W\right)^{2} \mid \omega_{i}\right]\right)=\frac{\left|K_{u}\right|^{2}}{|K|} \sum_{r=1}^{s} \frac{p_{2}\left(K_{r}\right)^{2}}{\left|K_{r}\right|}\left(\omega_{t}\left(g_{r}\right)+1-2 \omega_{t}\left(g_{u}\right)\right)^{2} .
$$

Note that the sum in the second part of the lemma begins at $r=1$ and so excludes the $r=0$ term which corresponds to the trivial spherical function.

Proof. Observe that

$$
\begin{aligned}
\mathbb{E}\left(\left(W^{\prime}\right)^{2} \mid \omega_{i}\right) & =\frac{\left|K_{u}\right|}{|K|} \sum_{j=0}^{s} \frac{d_{j}}{|G|} \sum_{r=0}^{s}\left|K_{r}\right| \omega_{i}\left(g_{r}\right) \omega_{t}\left(g_{r}\right) \overline{\omega_{j}\left(g_{r}\right)} \omega_{j}\left(g_{u}\right)^{2} \\
& =\frac{\left|K_{u}\right|}{|K|} \sum_{r=0}^{s}\left|K_{r}\right| \omega_{i}\left(g_{r}\right) \omega_{t}\left(g_{r}\right) \frac{1}{|G|} \sum_{j=0}^{s} d_{j} \omega_{j}\left(g_{u}\right)^{2} \overline{\omega_{j}\left(g_{r}\right)} \\
& =\frac{\left|K_{u}\right|}{|K|} \sum_{r=0}^{s} \omega_{i}\left(g_{r}\right) \omega_{t}\left(g_{r}\right) p_{2}\left(K_{r}\right) .
\end{aligned}
$$

The second equality switched the order of summation, and the final equality was Lemma 4.4 .

Next we claim that $W^{2}=\frac{\left|K_{u}\right|}{|K|} \sum_{r=0}^{s} p_{2}\left(K_{r}\right) \omega_{i}\left(g_{r}\right)$. To see this note by Lemma 4.4 that

$$
\left|K_{r}\right| \sum_{j=0}^{s} \frac{d_{j}}{|G|} \omega_{j}\left(g_{u}\right)^{2} \overline{\omega_{j}\left(g_{r}\right)}=p_{2}\left(K_{r}\right) .
$$

Multiplying both sides by $\frac{\left|K_{u}\right|}{|K|} \omega_{i}\left(g_{r}\right)$ and summing over $r$, the claimed expression for $W^{2}$ follows from Lemma 4.2

To prove the first part of the lemma, note that Lemma 4.7 gives that

$$
\begin{aligned}
\mathbb{E}\left[\left(W^{\prime}-W\right)^{2} \mid \omega_{i}\right] & =\mathbb{E}\left(\left(W^{\prime}\right)^{2} \mid \omega_{i}\right)-2 W \mathbb{E}\left(W^{\prime} \mid \omega_{i}\right)+W^{2} \\
& =\mathbb{E}\left(\left(W^{\prime}\right)^{2} \mid \omega_{i}\right)+\left(1-2 \omega_{t}\left(g_{u}\right)\right) W^{2} .
\end{aligned}
$$

Now use the previous two paragraphs.

The first part of the lemma and Lemma 4.3 imply that

$$
\mathbb{E}\left(\mathbb{E}\left[\left(W^{\prime}-W\right)^{2} \mid \omega_{i}\right]^{2}\right)=\frac{\left|K_{u}\right|^{2}}{|K|} \sum_{r=0}^{s} \frac{p_{2}\left(K_{r}\right)^{2}}{\left|K_{r}\right|}\left(\omega_{t}\left(g_{r}\right)+1-2 \omega_{t}\left(g_{u}\right)\right)^{2} .
$$

From Lemmas 4.4 and 4.3, $p_{2}(K)=\frac{|K|}{\left|K_{\mu}\right|}$, so the $r=0$ term contributes $4\left(1-\omega_{t}\left(g_{u}\right)\right)^{2}$. The second part of the lemma follows since by Lemma 4.9 .

$$
\operatorname{Var}\left(\mathbb{E}\left[\left(W^{\prime}-W\right)^{2} \mid \omega_{i}\right]\right)=\mathbb{E}\left(\mathbb{E}\left[\left(W^{\prime}-W\right)^{2} \mid \omega_{i}\right]^{2}\right)-4\left(1-\omega_{t}\left(g_{u}\right)\right)^{2} .
$$

Lemma 4.11. Let $k$ be a positive integer.

(1) $\mathbb{E}\left(W^{\prime}-W\right)^{k}$ is equal to

$$
\left(\frac{\left|K_{u}\right|}{|K|}\right)^{k / 2} \sum_{m=0}^{k}(-1)^{k-m}\left(\begin{array}{c}
k \\
m
\end{array}\right) \sum_{r=0}^{s} \frac{|K|}{\left|K_{r}\right|} \omega_{t}\left(g_{r}\right) p_{m}\left(K_{r}\right) p_{k-m}\left(K_{r}\right) .
$$

(2) $\mathbb{E}\left(W^{\prime}-W\right)^{4}=\frac{\left|K_{u}\right|^{2}}{|K|} \sum_{r=0}^{s}\left[8\left(1-\omega_{t}\left(g_{u}\right)\right)-6\left(1-\omega_{t}\left(g_{r}\right)\right)\right] \frac{p_{2}\left(K_{r}\right)^{2}}{\left|K_{r}\right|}$. 
Proof. For the first assertion, note that $\mathbb{E}\left(\left(W^{\prime}-W\right)^{k} \mid \omega_{i}\right)$ is equal to

$$
\begin{aligned}
& \left(\frac{\left|K_{u}\right|}{|K|}\right)^{k / 2} \sum_{j=0}^{s} \frac{d_{j}}{|G|} \sum_{r=0}^{s}\left|K_{r}\right| \omega_{i}\left(g_{r}\right) \omega_{t}\left(g_{r}\right) \overline{\omega_{j}\left(g_{r}\right)} \\
& \cdot \sum_{m=0}^{k}(-1)^{k-m}\left(\begin{array}{c}
k \\
m
\end{array}\right) \omega_{j}\left(g_{u}\right)^{m} \omega_{i}\left(g_{u}\right)^{k-m} \\
= & \left(\frac{\left|K_{u}\right|}{|K|}\right)^{k / 2} \sum_{m=0}^{k}(-1)^{k-m}\left(\begin{array}{c}
k \\
m
\end{array}\right) \omega_{i}\left(g_{u}\right)^{k-m} \\
& \cdot \sum_{r=0}^{s}\left|K_{r}\right| \omega_{i}\left(g_{r}\right) \omega_{t}\left(g_{r}\right) \sum_{j=0}^{s} \frac{d_{j}}{|G|} \omega_{j}\left(g_{u}\right)^{m} \overline{\omega_{j}\left(g_{r}\right)} \\
= & \left(\frac{\left|K_{u}\right|}{|K|}\right)^{k / 2} \sum_{m=0}^{k}(-1)^{k-m}\left(\begin{array}{c}
k \\
m
\end{array}\right) \omega_{i}\left(g_{u}\right)^{k-m} \sum_{r=0}^{s} \omega_{i}\left(g_{r}\right) \omega_{t}\left(g_{r}\right) p_{m}\left(K_{r}\right) .
\end{aligned}
$$

The first equality switched the order of summation and the last equality is Lemma 4.4. Hence

$$
\begin{aligned}
\mathbb{E}\left(W^{\prime}-W\right)^{k}= & \mathbb{E}\left(\mathbb{E}\left(\left(W^{\prime}-W\right)^{k} \mid \omega_{i}\right)\right) \\
= & \left(\frac{\left|K_{u}\right|}{|K|}\right)^{k / 2} \sum_{m=0}^{k}(-1)^{k-m}\left(\begin{array}{c}
k \\
m
\end{array}\right) \\
& \cdot \sum_{r=0}^{s} \omega_{t}\left(g_{r}\right) p_{m}\left(K_{r}\right) \sum_{i=0}^{s} \frac{d_{i}|K|}{|G|} \omega_{i}\left(g_{r}\right) \omega_{i}\left(g_{u}\right)^{k-m} .
\end{aligned}
$$

The first assertion follows from Lemma 4.4 and the fact that $\omega_{i}\left(g_{u}\right)$ is real.

For the second assertion, one knows from the first assertion that

$$
\mathbb{E}\left(W^{\prime}-W\right)^{4}=\left(\frac{\left|K_{u}\right|}{|K|}\right)^{2} \sum_{m=0}^{4}(-1)^{m}\left(\begin{array}{c}
4 \\
m
\end{array}\right) \sum_{r=0}^{s} \frac{|K|}{\left|K_{r}\right|} \omega_{t}\left(g_{r}\right) p_{m}\left(K_{r}\right) p_{4-m}\left(K_{r}\right) .
$$

The special case $t=0$ gives the equation

$$
0=\left(\frac{\left|K_{u}\right|}{|K|}\right)^{2} \sum_{m=0}^{4}(-1)^{m}\left(\begin{array}{c}
4 \\
m
\end{array}\right) \sum_{r=0}^{s} \frac{|K|}{\left|K_{r}\right|} p_{m}\left(K_{r}\right) p_{4-m}\left(K_{r}\right) .
$$

Hence in general, $\mathbb{E}\left(W^{\prime}-W\right)^{4}$ is equal to

$$
-\frac{\left|K_{u}\right|^{2}}{|K|^{2}} \sum_{m=0}^{4}(-1)^{m}\left(\begin{array}{c}
4 \\
m
\end{array}\right) \sum_{r=0}^{s} \frac{|K|}{\left|K_{r}\right|}\left(1-\omega_{t}\left(g_{r}\right)\right) p_{m}\left(K_{r}\right) p_{4-m}\left(K_{r}\right) .
$$

The $m=0,4$ terms both vanish since $p_{0}\left(K_{r}\right)=0$ if $r \neq 0$. The $m=2$ term is equal to

$$
-\frac{6\left|K_{u}\right|^{2}}{|K|} \sum_{r=0}^{s} \frac{\left(1-\omega_{t}\left(g_{r}\right)\right) p_{2}\left(K_{r}\right)^{2}}{\left|K_{r}\right|}
$$


The $m=1,3$ terms are equal and together contribute

$$
\begin{aligned}
\frac{8\left|K_{u}\right|^{2}}{|K|} \sum_{r=0}^{s} \frac{\left(1-\omega_{t}\left(g_{r}\right)\right) p_{1}\left(K_{r}\right) p_{3}\left(K_{r}\right)}{\left|K_{r}\right|} & =\frac{8\left|K_{u}\right|}{|K|}\left(1-\omega_{t}\left(g_{u}\right)\right) p_{3}\left(K_{u}\right) \\
& =\frac{8\left|K_{u}\right|^{2}}{|K|^{2}}\left(1-\omega_{t}\left(g_{u}\right)\right) p_{4}\left(K_{0}\right) \\
& =\frac{8\left|K_{u}\right|^{2}}{|K|}\left(1-\omega_{t}\left(g_{u}\right)\right) \sum_{r=0}^{s} \frac{p_{2}\left(K_{r}\right)^{2}}{\left|K_{r}\right|} .
\end{aligned}
$$

The last two equations can be seen directly or from Lemma 4.4. This completes the proof of the second assertion.

Arguing as in the proof of Theorem [3.12, and using the above lemmas, one obtains the following result.

Theorem 4.12. Let $(G, K)$ be a Gelfand pair, and fix a double coset $K_{u}=K g_{u} K$ of $G$ satisfying $K g_{u} K=K g_{u}^{-1} K$. Let $\omega_{t}$ be a real valued spherical function such that $0<\omega_{t}\left(g_{u}\right)<1$. Choosing $\omega_{i}$ from the Plancherel measure of the pair $(G, K)$, let $W=\frac{\left|K_{u}\right|^{1 / 2}}{|K|^{1 / 2}} \omega_{i}\left(g_{u}\right)$. Then for all real $x_{0}$,

$$
\begin{aligned}
& \left|\mathbb{P}\left(W \leq x_{0}\right)-\frac{1}{\sqrt{2 \pi}} \int_{-\infty}^{x_{0}} e^{-\frac{x^{2}}{2}} d x\right| \\
\leq & \frac{\left|K_{u}\right|}{a|K|} \sqrt{\sum_{r=1}^{s} \frac{|K| p_{2}\left(K_{r}\right)^{2}}{\left|K_{r}\right|}\left(\omega_{t}\left(g_{r}\right)+2 a-1\right)^{2}} \\
& +\frac{1}{(\pi)^{1 / 4}} \frac{\left|K_{u}\right|^{1 / 2}}{|K|^{1 / 2}}\left[\sum_{r=0}^{s}\left(8-\frac{6\left(1-\omega_{t}\left(g_{r}\right)\right)}{a}\right) \frac{|K| p_{2}\left(K_{r}\right)^{2}}{\left|K_{r}\right|}\right]^{1 / 4},
\end{aligned}
$$

where $a=1-\omega_{t}\left(g_{u}\right)$.

4.3. Example: The hypercube. The $n$ dimensional hypercube $\mathbb{Z}_{2}^{n}$ consists of $n$ tuples of 0 's and 1's. Random walk on it proceeds by picking a random coordinate and changing it. The spectrum of this random walk is well known; for each $0 \leq i \leq n$ there is an eigenvalue $1-\frac{2 i}{n}$ occurring with multiplicity $\left(\begin{array}{c}n \\ i\end{array}\right)$ (see for instance page 28 of [8]). Thus by the usual central limit theorem for the binomial distribution, the spectrum of the hypercube is asymptotically normal with an error term $O\left(n^{-1 / 2}\right)$. The purpose of this subsection is to revisit this classical result from the viewpoint of Gelfand pairs, illustrating the construction of Subsection 4.2 .

To begin, note from the remarks on page 58 of [8] that the hypercube can be viewed as $G / K$ for a certain Gelfand pair $(G, K)$. Namely $G$ is the semidirect product of $\mathbb{Z}_{2}^{n}$ with $S_{n}$, where the group multiplication is $(x, \pi)(y, \tau)=(x+\pi(y), \pi \tau)$, where $\pi(y)$ permutes the coordinates of $y$. $K$ is the subgroup $\left\{(0, \pi): \pi \in S_{n}\right\}$. The induced module $1_{K}^{G}$ decomposes as $\bigoplus_{r=0}^{n} V_{r}$ where $d_{r}=\left(\begin{array}{c}n \\ r\end{array}\right)$. Thus the Plancherel measure chooses $i \in\{0, \cdots, n\}$ with probability $\frac{\left(\begin{array}{c}n \\ i\end{array}\right)}{2^{n}}$. For $0 \leq r \leq n$, the double coset $K_{r}$ in $G$ consists of elements $(x, \pi)$ where $x$ has $r$ coordinates equal to 1 . Thus $\left|K_{r}\right|=\left(\begin{array}{l}n \\ r\end{array}\right) n$ !, and as usual let $g_{r}$ denote some element of $K_{r}$. The spherical 
function $\omega_{i}(0 \leq i \leq n)$ is given by

$$
\omega_{i}\left(g_{r}\right)=\frac{1}{\left(\begin{array}{c}
n \\
i
\end{array}\right)} \sum_{m=0}^{i}(-1)^{m}\left(\begin{array}{c}
r \\
m
\end{array}\right)\left(\begin{array}{c}
n-r \\
i-m
\end{array}\right)
$$

which is a Krawtchouk polynomial if one overlooks the $\left(\begin{array}{c}n \\ i\end{array}\right)$ in the denominator.

The following result emerges from Theorem 4.12. Since $\omega_{i}\left(g_{1}\right)=1-\frac{2 i}{n}$ (or by Lemma 4.5), it can be seen as a central limit theorem for the spectrum of random walk on the hypercube.

Theorem 4.13. Let $W=\sqrt{n} \omega_{i}\left(g_{1}\right)$ where $i$ is chosen from Plancherel measure. Then for all real $x_{0}$,

$$
\left|\mathbb{P}\left(W \leq x_{0}\right)-\frac{1}{\sqrt{2 \pi}} \int_{-\infty}^{x_{0}} e^{-\frac{x^{2}}{2}} d x\right| \leq\left(\frac{8}{\pi n}\right)^{1 / 4}
$$

Proof. Apply Theorem 4.12 with $u=1$ and $t=1$. Then $a=\frac{2}{n}$. Note that $p_{2}\left(K_{r}\right)$ is the chance that random walk on the hypercube started at the vertex with all coordinates 0 will have $r$ coordinates equal to 1 after 2 steps. Thus $p_{2}\left(K_{0}\right)=\frac{1}{n}$, $p_{2}\left(K_{2}\right)=1-\frac{1}{n}$, and $p_{2}\left(K_{r}\right)=0$ for all $r \neq 0,2$. Since $\omega_{1}\left(g_{2}\right)=1-\frac{4}{n}$, one has that $\omega_{1}\left(g_{2}\right)+2 a-1=0$. Thus the first error term in Theorem 4.12 is 0 . The second error term in Theorem 4.12 is computed to be $\left(\frac{8}{\pi n}\right)^{1 / 4}$, implying the result.

To get $O\left(n^{-1 / 2}\right)$ bounds by Stein's method, it will be shown that $\left|W^{\prime}-W\right|$ is bounded, so that one can use the version of Stein's method in Theorem 2.2 instead of that in Theorem 2.1. The boundedness of $\left|W^{\prime}-W\right|$ will follow from Lemma 4.14, which proves that $L_{1}$ is in fact a birth-death chain.

Lemma 4.14. The chain $L_{1}$ on the set $\{0, \cdots, n\}$ is a birth-death chain with transition probabilities

$$
L_{1}(i, j)= \begin{cases}\frac{i}{n} & \text { if } j=i-1 \\ 1-\frac{i}{n} & \text { if } j=i+1\end{cases}
$$

Proof. From the three term recurrence for Krawtchouk polynomials on page 152 of 30, it follows that

$$
(i+1)\left(\begin{array}{c}
n \\
i+1
\end{array}\right) \omega_{i+1}\left(g_{r}\right)=(n-2 r)\left(\begin{array}{c}
n \\
i
\end{array}\right) \omega_{i}\left(g_{r}\right)-(n-i+1)\left(\begin{array}{c}
n \\
i-1
\end{array}\right) \omega_{i-1}\left(g_{r}\right)
$$

Since $n-2 r=n \omega_{1}\left(g_{r}\right)$, one obtains that

$$
n\left(\begin{array}{c}
n \\
i
\end{array}\right) \omega_{1}\left(g_{r}\right) \omega_{i}\left(g_{r}\right)=(i+1)\left(\begin{array}{c}
n \\
i+1
\end{array}\right) \omega_{i+1}\left(g_{r}\right)+(n-i+1)\left(\begin{array}{c}
n \\
i-1
\end{array}\right) \omega_{i-1}\left(g_{r}\right) .
$$

Simplifying, one obtains the relation

$$
\omega_{1}\left(g_{r}\right) \omega_{i}\left(g_{r}\right)=\left(1-\frac{i}{n}\right) \omega_{i+1}\left(g_{r}\right)+\left(\frac{i}{n}\right) \omega_{i-1}\left(g_{r}\right) .
$$

The result now follows from Lemma 4.2 and the definition of $L_{1}$.

Now an $O\left(n^{-1 / 2}\right)$ error term is established. 
Theorem 4.15. Let $W=\sqrt{n} \omega_{i}\left(g_{1}\right)$ where $i$ is chosen from Plancherel measure. Then for all real $x_{0}$,

$$
\left|\mathbb{P}\left(W \leq x_{0}\right)-\frac{1}{\sqrt{2 \pi}} \int_{-\infty}^{x_{0}} e^{-\frac{x^{2}}{2}} d x\right| \leq 5 n^{-1 / 2} .
$$

Proof. Apply the variation of Theorem 4.12 which would arise from using Theorem 2.2 instead of Theorem 2.1. Recall that $a=\frac{2}{n}$. Also $\left|W^{\prime}-W\right| \leq A$ with $A=$ $\frac{2}{\sqrt{n}}$ since $L_{1}$ is a birth-death chain. As explained in the proof of Theorem 4.13, $\operatorname{Var}\left(\mathbb{E}\left[\left(W^{\prime}-W\right)^{2} \mid W\right]\right)=0$. The result follows.

As a final remark, Lemma 4.14 shows that $L_{1}$ is closely related to the birth-death chain used by Stein 41 in proving a central limit theorem for $X_{1}+\ldots+X_{n}$, where the $X$ 's are independent and each equal to 0 or 1 with probability $1 / 2$. To form an exchangeable pair Stein chose a random $l \in\{1, \cdots, n\}$ and then replaced $X_{l}$ by a new random variable which is also 0 or 1 with probability $1 / 2$ and independent of all of the $X$ 's. The chain $L_{1}$ would arise by picking a random index $l$ and switching the value of $X_{l}$.

4.4. Example: Random walk on perfect matchings. In this example $G=S_{2 n}$ and $K$ is the hyperoctahedral group of signed permutations on $n$ symbols, of size $2^{n} n !$. This Gelfand pair is discussed at length in Chapter 7 of 29] and in Section 3 of [19], to which we refer the reader for proofs of facts in the next two paragraphs. Another useful reference is 9 .

The induced representation $1_{K}^{G}$ decomposes as $\bigoplus_{|\lambda|=n} V_{\lambda}$, where $\lambda$ ranges over all partitions of size $n$. An explicit formula for the numbers $d_{\lambda}$ appears in [29] and is not needed in what follows. However it is worth remarking that the Plancherel measure of $(G, K)$, which chooses $\lambda$ with probability $\frac{2^{n} n ! d_{\lambda}}{(2 n) !}$, is the $\alpha=2$ case of the so called $\mathrm{Jack}_{\alpha}$ measure on partitions, which chooses $\lambda$ with probability

$$
\frac{\alpha^{n} n !}{\prod_{s \in \lambda}(\alpha a(s)+l(s)+1)(\alpha a(s)+l(s)+\alpha)}
$$

where the product is over all boxes of the partition. Here $a(s)$ is the number of boxes in the same row of $s$ and to the right of $s$ (the "arm" of $s$ ), and $l(s)$ is the number of boxes in the same column of $s$ and below $s$ (the "leg" of $s$ ). For example the partition of 5 below

would have $\mathrm{Jack}_{\alpha}$ measure

$$
\frac{60 \alpha^{2}}{(2 \alpha+2)(3 \alpha+1)(\alpha+2)(2 \alpha+1)(\alpha+1)} .
$$

The Jack measure on partitions is of interest to researchers in random matrix theory [4], [33, 27].

The double cosets $K_{\mu}$ of $K$ in $G$ are parameterized by partitions $\mu$ of size $n$ and have the following concrete description. A perfect matching of $\{1, \cdots, 2 n\}$ can be regarded as a 1-regular graph with vertex set $\{1, \cdots, 2 n\}$. Let $\epsilon$ be the "identity matching" in which $i$ is adjacent to $n+i$ for $1 \leq i \leq n$. Given a permutation $w$ in $S_{2 n}$, let $\delta(w)$ be the perfect matching of $\{1, \cdots, 2 n\}$ in which $i$ is adjacent to $j$ if and only $|w(i)-w(j)|=n$. For example, $\delta(i d)=\epsilon$. Note that the union $\delta_{1} \cup \delta_{2}$ of two 1-regular graphs is a 2-regular graph, and thus a disjoint union of even length 
cycles. Let $\Lambda\left(\delta_{1}, \delta_{2}\right)$ be the partition of $n$ whose parts are half the cycle lengths of $\delta_{1} \cup \delta_{2}$. Then $K w_{1} K=K w_{2} K$ if and only if $\Lambda\left(\epsilon, \delta\left(w_{1}\right)\right)=\Lambda\left(\epsilon, \delta\left(w_{2}\right)\right)$. Thus $K w^{-1} K=K w K$ for all $w$, so that the machinery of Subsection 4.2 is applicable. One also has that $K w_{1}=K w_{2}$ if and only if $\delta\left(w_{1}\right)=\delta\left(w_{2}\right)$. It follows that $G / K$ is in bijection with the perfect matchings of $\{1, \cdots, 2 n\}$ and that $\frac{\left|K_{\mu}\right|}{|K|}$ is equal to the number of perfect matchings $\delta$ with $\Lambda(\epsilon, \delta)=\mu$, which by elementary counting is $\frac{2^{n} n !}{2^{l(\mu)} \prod_{j} m_{j}(\mu) ! j^{m_{j}(\mu)}}$ where $l(\mu)$ is the number of parts of $\mu$ and $m_{j}(\mu)$ is the number of parts of $\mu$ of size $j$.

The main purpose of this subsection is to prove a central limit theorem for the random variable $W=\sqrt{2^{i-1}\left(\begin{array}{c}n \\ i\end{array}\right)(i-1) !} \omega_{\lambda}\left(g_{\left(i, 1^{n-i}\right)}\right)$, where $i$ is fixed and $\lambda$ is chosen from the Plancherel measure of $(G, K)$. Then Lemma 4.5 gives a central limit theorem for the spectrum of random walk on the graph $H_{\left(i, 1^{n-i}\right)}$, whose vertices are the perfect matchings of $\{1, \cdots, 2 n\}$, with an edge between matchings $\delta_{1}$ and $\delta_{2}$ if and only if $\Lambda\left(\delta_{1}, \delta_{2}\right)=\left(i, 1^{n-i}\right)$. In the case $i=2$, the spectrum of this graph was determined in [9], and was shown to be asymptotically normal in 15] (with error term $O\left(n^{-1 / 4}\right)$ ) and in [16] (with error term $O\left(n^{-1 / 2}\right)$ ). But the arguments in those papers used different information, and it was not clear that they could be pushed through to larger $i$. Theorem 4.12 will be used to deduce a central limit theorem for any fixed $i$ with error term $O\left(n^{-1 / 4}\right)$.

To apply Theorem 4.12 $\omega_{t}$ will be taken to be the spherical function $\omega_{(n-1,1)}$. An explicit formula for $\omega_{(n-1,1)}$ is available.

Lemma 4.16 ([29], p. 411). Let $m_{1}(\mu)$ denote the number of parts of size 1 of $\mu$. Then

$$
\omega_{(n-1,1)}\left(g_{\mu}\right)=\frac{(2 n-1) m_{1}(\mu)-n}{2 n(n-1)}
$$

for all $\mu$ of size $n$.

Lemma 4.17 is helpful.

Lemma 4.17 ([19, Lemma 3.2). The coefficient of $K_{\mu}$ in $K_{\tau} K_{\left(i, 1^{n-i}\right)}$ is equal to $\frac{|K|^{2}}{K_{\mu}}$ multiplied by the number of pairs of perfect matchings $(\delta, \gamma)$ such that $\Lambda(\epsilon, \delta)=$ $\tau, \Lambda(\delta, \gamma)=\left(i, 1^{n-i}\right)$, and $\Lambda(\epsilon, \gamma)=\mu$.

The final combinatorial ingredient is an analog of Lemma 3.13

Lemma 4.18. Consider the random walk on $G$ generated by $K_{\left(i, 1^{n-i}\right)}$. Then for $i$ fixed and $n \geq 2 i, \frac{p_{2}\left(K_{\mu}\right)^{2}|K|}{\left|K_{\mu}\right|}$ is equal to

(1) $\frac{i^{2}}{4^{i-1}} n^{-2 i}+O\left(n^{-2 i-1}\right)$ if $K_{\mu}=K_{\left(1^{n}\right)}=K$.

(2) $\frac{2 i^{2}}{4^{i-1}} n^{-2 i}+O\left(n^{-2 i-1}\right)$ if $K_{\mu}=K_{\left(i, i, 1^{n-2 i}\right)}$.

(3) $O\left(n^{-2 i-1}\right)$ otherwise.

Proof. Lemma 4.17 implies that $\frac{p_{2}\left(K_{\mu}\right)\left|K_{\left(i, 11^{n-i}\right)}\right|^{2}}{|K|^{2}}$ is equal to the number of pairs of matchings $(\delta, \gamma)$ such that $\Lambda(\epsilon, \delta)=\left(i, 1^{n-i}\right), \Lambda(\delta, \gamma)=\left(i, 1^{n-i}\right)$, and $\Lambda(\epsilon, \gamma)=\mu$.

For the first assertion, it follows either from the previous paragraph or from Lemmas 4.4 and 4.3 that $p_{2}(K)=\frac{1}{2^{i-1}\left(\begin{array}{c}n \\ i\end{array}\right)(i-1) !}$. For the second assertion, it is straightforward from the previous paragraph that $p_{2}\left(K_{\left(i, i, 1^{n-2 i}\right)}\right)=1+O\left(n^{-1}\right)$. Then use the formula for $\frac{\left|K_{\mu}\right|}{|K|}$ given earlier in this subsection. 
For the third assertion, there are two cases. The first case is that $\mu$ has $n-2 i$ parts of size 1 , but is not equal to $\left(i, i, 1^{n-2 i}\right)$. Then $p_{2}\left(K_{\mu}\right)=O\left(n^{-1}\right)$ and the formula for $\frac{|K|}{\left|K_{\mu}\right|}$ shows it to be at most $c_{i} n^{-2 i}$ where $c_{i}$ is a constant depending on $i$. So in the first case, the result is proved. The second case is that $\mu$ has $n-2 i+r$ parts of size 1 , where $1 \leq r<2 i$. From the first paragraph of the proof, it is straightforward to see that $p_{2}\left(K_{\mu}\right)=O\left(n^{-r}\right)$. Also the formula for $\frac{|K|}{\left|K_{\mu}\right|}$ shows it to be $O\left(n^{r-2 i}\right)$, proving the result.

Combining the ingredients, one deduces the following result.

Theorem 4.19. Choose $\lambda$ from the Plancherel measure of the Gelfand pair $(G, K)$, and define a random variable $W=\sqrt{2^{i-1}\left(\begin{array}{c}n \\ i\end{array}\right)(i-1) !} \omega_{\lambda}\left(g_{\left(i, 1^{n-i}\right)}\right)$. Then there is a constant $A_{i}$ such that for all real $x_{0}$,

$$
\left|\mathbb{P}\left(W \leq x_{0}\right)-\frac{1}{\sqrt{2 \pi}} \int_{-\infty}^{x_{0}} e^{-\frac{x^{2}}{2}} d x\right| \leq A_{i} n^{-1 / 4} .
$$

Proof. One applies Theorem 4.12 choosing $u=\left(i, 1^{n-i}\right)$ and $\omega_{t}$ to be the spherical function $\omega_{(n-1,1)}$. By Lemma 4.16, $\omega_{t}$ is real valued and $a=\frac{i(2 n-1)}{2 n(n-1)}$. Observe that $\omega_{t}\left(g_{\mu}\right)+2 a-1$ vanishes if $\mu=\left(i, i, 1^{n-2 i}\right)$ and by Lemma 4.16 is $O\left(n^{-1}\right)$ for any other $\mu$ such that $p_{2}\left(K_{\mu}\right) \neq 0$, since any such $\mu$ has at least $n-2 i$ parts of size 1 . Thus by Lemma 4.18 .

$$
\sum_{\mu \neq\left(1^{n}\right)} \frac{|K| p_{2}\left(K_{\mu}\right)^{2}}{\left|K_{\mu}\right|}\left(\omega_{t}\left(g_{\mu}\right)+2 a-1\right)^{2}=O\left(n^{-2 i-3}\right) .
$$

Since $\frac{\left|K_{u}\right|}{|K|}=2^{i-1}\left(\begin{array}{c}n \\ i\end{array}\right)(i-1)$ !, the first error term in Theorem4.12 is at most $A_{i}^{\prime} n^{-1 / 2}$, where $A_{i}^{\prime}$ is a constant depending on $i$.

To bound the second error term in Theorem 4.12, we claim that

$$
\sum_{\mu}\left(8-\frac{6\left(1-\omega_{t}\left(g_{\mu}\right)\right)}{a}\right) \frac{|K| p_{2}\left(K_{\mu}\right)^{2}}{\left|K_{\mu}\right|}=O\left(n^{-2 i-1}\right) .
$$

Indeed, by Lemmas 4.16 and 4.18, the term $\mu=\left(1^{n}\right)$ contributes $\frac{8 i^{2}}{4^{i-1}} n^{-2 i}+$ $O\left(n^{-2 i-1}\right)$ and the term $\mu=\left(i, i, 1^{n-2 i}\right)$ contributes $-\frac{8 i^{2}}{4^{i-1}} n^{-2 i}+O\left(n^{-2 i-1}\right)$. These lemmas also show that all other terms coming from a $\mu$ with $p_{2}\left(K_{\mu}\right) \neq 0$ contribute $O\left(n^{-2 i-1}\right)$, and the number of such $\mu$ is bounded by a constant depending on $i$. It follows that the second error term is at most $A_{i}^{\prime \prime} n^{-1 / 4}$, where $A_{i}^{\prime \prime}$ is another constant depending only on $i$.

\section{Twisted Gelfand PAIRS}

The approach taken in this section is a bit different than that in the sections on finite groups and Gelfand pairs. Due both to a lack of interesting examples of twisted Gelfand pairs which are not already Gelfand pairs and to technical complications which did not arise in earlier sections of this paper (see the discussion in Section 5.2 for details), a completely general theory is not developed. Instead, we focus on one very interesting example: character values of random projective representations of the symmetric group. It should however be noted that many of the lemmas resemble those of earlier sections, and the calculations are organized in a way which should generalize to other examples. 
A partition $\lambda$ of $n$ is called strict if all of its parts are distinct, and is called odd if all of its parts are odd. It will be useful to have the notation that $D P(n)$ is the set of strict partitions of size $n$ and $O P(n)$ is the set of odd partitions of size $n$.

This section uses Stein's method to study the probability measure on strict partitions of $n$ which chooses $\lambda$ with probability

$$
\frac{2^{n-l(\lambda)} g_{\lambda}^{2}}{n !}
$$

where $l(\lambda)$ is the number of parts of a partition $\lambda$ and $g_{\lambda}$ is the number of standard shifted tableaux of shape $\lambda([20,[29])$. This measure on strict partitions is known as shifted Plancherel measure, and is of interest to researchers in random matrix theory [31, [45].

Ivanov 23] studied character values of random projective representations of the symmetric group. It is known (see 20] for a friendly exposition) that the character values in question are expressed in terms of the coefficients $X_{\rho}^{\lambda}$, where $\lambda$ is a strict partition of $n$ that parameterizes an irreducible character, and $\mu$ is an odd partition of $n$ that parameterizes a conjugacy class. One also has that $g_{\lambda}=X_{\left(1^{n}\right)}^{\lambda}$. Ivanov proved the following central limit theorem for these character values.

Theorem 5.1 (23). Fix $i \geq 1$. Let $\lambda$ be chosen from the shifted Plancherel measure on strict partitions of size $n$. Then as $n \rightarrow \infty$, the random variable $\frac{n^{\frac{2 i+1}{2}} X_{(2 i+1,1}^{\lambda-2 i-1)}}{2^{i} \sqrt{2 i+1} g_{\lambda}}$ converges in distribution to a normal random variable with mean 0 and variance 1 .

This section refines the result of Ivanov, which was proved by the method of moments, so as to obtain an error term. In the statement of the result, recall that $z_{\mu}=\prod_{j \geq 1} j^{m_{j}(\mu)} m_{j}(\mu)$ !, where $m_{j}(\mu)$ is the number of parts of $\mu$ of size $j$.

Theorem 5.2. Fix $i \geq 1$ and let $\mu=\left(2 i+1,1^{n-2 i-1}\right)$. Choosing $\lambda$ from the shifted Plancherel measure on partitions of size $n$, define a random variable

$$
W=\sqrt{\frac{n !}{z_{\mu} 2^{n-l(\mu)}}} \frac{X_{\mu}^{\lambda}}{g_{\lambda}} .
$$

Then there is a constant $A_{i}$ such that for all real $x_{0}$,

$$
\left|\mathbb{P}\left(W \leq x_{0}\right)-\frac{1}{\sqrt{2 \pi}} \int_{-\infty}^{x_{0}} e^{-\frac{x^{2}}{2}} d x\right| \leq A_{i} n^{-1 / 4} .
$$

The organization of this section is as follows. Subsection 5.1 defines twisted Gelfand pairs and collects and develops facts about their representation theory. Subsection 5.2 defines and studies a Markov chain to be used in the construction of an exchangeable pair for a Stein's method proof of Theorem 5.2. This is more subtle than the corresponding treatment in earlier sections and involves interesting combinatorics, since the "obvious" adaptation of the construction for Gelfand pairs does not work. Subsection 5.3 studies the exchangeable pair arising from the Markov chain in Section 5.2, and uses it to prove Theorem 5.2.

5.1. Background from representation theory. If $G$ is a finite group, $K$ a subgroup of $G$, and $\phi$ a linear character of $K$ such that $\operatorname{Ind}_{K}^{G}(\phi)$ is multiplicity free, the triple $(G, K, \phi)$ is called a twisted Gelfand pair (this terminology was 
introduced in [42]). The Hecke algebra of the triple $(G, K, \phi)$ is the $\mathbb{C} G$ subalgebra $e \mathbb{C} G e$, where $e$ is the primitive idempotent of $\mathbb{C} K$ defined by

$$
e=\frac{1}{|K|} \sum_{k \in K} \phi\left(k^{-1}\right) k
$$

One reason that twisted Gelfand pairs are interesting is that the Hecke algebra of the triple $(G, K, \phi)$ is commutative. The paper [42] is a good reference for the theory of twisted Gelfand pairs.

The twisted Gelfand pair of interest to us is the one studied in [42. Thus $G=S_{2 n}$ and $K$ is the hyperoctahedral group $B_{n}$ of signed permutations, imbedded in $G$ as the centralizer of the involution $(1,2)(3,4) \cdots(2 n-1,2 n)$. To define $\phi$, note that $B_{n}$ is the semidirect product of the groups $T_{n}$ and $\Sigma_{n}$, where $T_{n}$ is the subgroup (isomorphic to $\mathbb{Z}_{2}^{n}$ ) generated by $(1,2), \cdots,(2 n-1,2 n)$ and $\Sigma_{n}$ is the subgroup (isomorphic to $S_{n}$ ) generated by the "double transpositions" $(2 i-1,2 j-1)(2 i, 2 j)$ for $1 \leq i<j \leq n$. Then $\phi$ is the linear character of $B_{n}$ whose restriction to $\Sigma_{n}$ is the sign character, and whose restriction to $T_{n}$ is trivial.

Stembridge [42] defines twisted spherical functions for twisted Gelfand pairs (the analog of spherical functions for Gelfand pairs). One of his main results is that for $\left(S_{2 n}, B_{n}, \phi\right)$, their values are (aside from scalar multiples) equal to the $X_{\mu}^{\lambda}$, where $\lambda$ is a strict partition and $\mu$ is an odd partition.

Next we record orthogonality relations for the quantities $X_{\mu}^{\lambda}$. These orthogonality relations are a special case of more general orthogonality relations for coefficients of power sum symmetric functions in Hall-Littlewood polynomials, where the parameter $t$ in the Hall-Littlewood polynomial is -1 . What this paper calls $X_{\mu}^{\lambda}$ is written as $X_{\mu}^{\lambda}(-1)$ in Chapter 3 of [29].

Lemma 5.3 ([29], page 247). For $\lambda, \rho \in D P(n)$,

$$
\sum_{\mu \in O P(n)} \frac{2^{l(\mu)}}{z_{\mu}} X_{\mu}^{\lambda} X_{\mu}^{\rho}=\delta_{\lambda, \rho} 2^{l(\lambda)} .
$$

Lemma 5.4 ([29], page 247). For $\mu, \sigma \in O P(n)$,

$$
\sum_{\lambda \in D P(n)} \frac{1}{2^{l(\lambda)}} X_{\mu}^{\lambda} X_{\sigma}^{\lambda}=\delta_{\mu, \sigma} \frac{z_{\mu}}{2^{l(\mu)}} .
$$

As in Subsection 4.4, the double cosets of $B_{n}$ in $S_{2 n}$ are indexed by partitions $\nu$ of $n$. It is useful to specify representatives $w_{\nu}$. For the case $\nu=(n)$, one defines

$$
w_{(n)}=(1,2, \cdots, 2 n),
$$

and for the general case $\nu=\left(\nu_{1}, \cdots, \nu_{l}\right)$, one defines

$$
\omega_{\nu}=w_{\left(\nu_{1}\right)} \circ \cdots \circ w_{\left(\nu_{l}\right)}
$$

where the operation $x \circ y$ (for $x \in S_{2 i}, y \in S_{2 j}$ ) denotes the embedding of $S_{2 i} \times S_{2 j}$ in $S_{2 i+2 j}$ with $S_{2 i}$ acting on $\{1, \cdots, 2 i\}$ and $S_{2 j}$ acting on $\{2 i+1, \cdots, 2 i+2 j\}$.

For $\nu \in O P(n)$, define

$$
\tilde{K}_{\nu}=\frac{1}{\left|B_{n}\right|^{2}} \sum_{x_{1}, x_{2} \in B_{n}} \phi\left(x_{1} x_{2}\right) x_{1} w_{\nu} x_{2}
$$


Corollary 3.2 of [42] shows that if $\nu \notin O P(n)$, then $\tilde{K}_{\nu}=0$, and that $\left\{\tilde{K}_{\nu}: \nu \in\right.$ $O P(n)\}$ is a basis for the Hecke algebra of $\left(S_{2 n}, B_{n}, \phi\right)$. Thus for $\mu, \nu \in O P(n)$, it is natural to study the coefficient of $\tilde{K}_{\nu}$ in $\left(\tilde{K}_{\mu}\right)^{m}$. Lemma 5.5 gives a character theoretic expression for this coefficient and is analogous to Lemmas 3.4 and 4.4 .

Lemma 5.5. Suppose that $\mu_{1}, \cdots, \mu_{m}, \nu \in O P(n)$. Then the coefficient of $\tilde{K}_{\nu}$ in $\tilde{K}_{\mu_{1}} \cdots \tilde{K}_{\mu_{m}}$ is equal to

$$
\frac{2^{l\left(\mu_{1}\right)-n} \cdots 2^{l\left(\mu_{m}\right)-n}}{z_{\nu}} \sum_{\rho \in D P(n)} 2^{n-l(\rho)} g_{\rho}^{2} \frac{X_{\nu}^{\rho}}{g_{\rho}} \frac{X_{\mu_{1}}^{\rho}}{g_{\rho}} \cdots \frac{X_{\mu_{m}}^{\rho}}{g_{\rho}} .
$$

In particular, the coefficient of $\tilde{K}_{\nu}$ in $\left(\tilde{K}_{\mu}\right)^{m}$ is

$$
\frac{n !\left(2^{l(\mu)-n}\right)^{m}}{z_{\nu}} \mathbb{E}\left[\frac{X_{\nu}^{\rho}}{g_{\rho}}\left(\frac{X_{\mu}^{\rho}}{g_{\rho}}\right)^{m}\right],
$$

where $\rho$ is random from shifted Plancherel measure on partitions of size $n$.

Proof. The equality is immediate from the definition of shifted Plancherel measure, so it is enough to establish the first expression. Stembridge [42] provides a basis of orthogonal idempotents $\left\{E_{\rho}: \rho \in D P(n)\right\}$ of the Hecke algebra of $(G, H, \phi)$ in terms of the projective characters of the symmetric group. More precisely, it follows from Proposition 4.1 and Corollary 6.2 of [42] that one obtains such a basis of orthogonal idempotents by defining

$$
E_{\rho}=2^{n-l(\rho)} g_{\rho} \sum_{\mu \in O P(n)} \frac{X_{\mu}^{\rho}}{z_{\mu}} \tilde{K}_{\mu} .
$$

Multiplying both sides of this equation by $2^{l(\mu)-n} \frac{X_{\mu}^{\rho}}{g_{\rho}}$ and summing over all $\rho \in$ $D P(n)$, it follows from Lemma 5.4 that

$$
\tilde{K}_{\mu}=2^{l(\mu)-n} \sum_{\rho \in D P(n)} \frac{X_{\mu}^{\rho}}{g_{\rho}} E_{\rho} .
$$

Thus

$$
\tilde{K}_{\mu_{1}} \cdots \tilde{K}_{\mu_{m}}=2^{l\left(\mu_{1}\right)-n} \cdots 2^{l\left(\mu_{m}\right)-n} \sum_{\rho \in D P(n)} \frac{X_{\mu_{1}}^{\rho}}{g_{\rho}} \cdots \frac{X_{\mu_{m}}^{\rho}}{g_{\rho}} E_{\rho}
$$

The result now follows by the formula for $E_{\rho}$ in the previous paragraph.

Lemma 5.6 derives an explicit formula for $X_{\mu}^{(n-1,1)}$. This is crucial to our approach.

Lemma 5.6. Let $m_{1}(\mu)$ denote the number of parts of size 1 of $\mu$. Suppose that $n \geq 3$, so that $(n-1,1)$ is a strict partition. Then

$$
X_{\mu}^{(n-1,1)}=m_{1}(\mu)-2
$$

for all odd partitions $\mu$ of size $n$.

Proof. Recall the definition of power sum symmetric functions: for $i \geq 1$, one sets $p_{i}=\sum_{j} x_{j}^{i}$, and for $\mu$ a partition of $n$, one sets $p_{\mu}=\prod_{i} p_{i}^{m_{i}(\mu)}$. The argument also 
uses Schur's Q-functions (29], Sec. 3.8). More precisely, equation 7.5 on page 247 of 29] shows that for $\mu \in O P(n)$ and $\lambda \in D P(n)$, the coefficient of the power sum symmetric function $p_{\mu}$ in $Q_{\lambda}$ is equal to $\frac{2^{l(\mu)} X_{\mu}^{\lambda}}{z_{\mu}}$.

To proceed one needs an expression for $Q_{(n-1,1)}$. Using the notation that $\left[x^{n}\right] f(x)$ is the coefficient of $x^{n}$ in $f(x)$, one has by page 253 of [29] that

$$
\begin{aligned}
Q_{(n-1,1)} & =\left[t_{1}^{n-1} t_{2}\right]\left(\frac{1-t_{2} / t_{1}}{1+t_{2} / t_{1}}\right) \prod_{i=1}^{2} \prod_{j=1}^{\infty} \frac{1+t_{i} x_{j}}{1-t_{i} x_{j}} \\
& =\left[t_{1}^{n-1}\right] \prod_{j=1}^{\infty} \frac{1+t_{1} x_{j}}{1-t_{1} x_{j}}\left[t_{2}\right] \prod_{j=1}^{\infty} \frac{1+t_{2} x_{j}}{1-t_{2} x_{j}}-2\left[t_{1}^{n}\right] \prod_{j=1}^{\infty} \frac{1+t_{1} x_{j}}{1-t_{1} x_{j}} \\
& =2 p_{1} Q_{n-1}-2 Q_{n} .
\end{aligned}
$$

By page 248 of [29], $X_{\mu}^{n}=1$ for all $\mu \in O P(n)$. It follows from the first paragraph that for $\mu \in O P(n)$, the coefficient of $p_{\mu}$ in $Q_{n}$ is $\frac{2^{l(\mu)}}{z_{\mu}}$ and (by considering separately the cases that $m_{1}(\mu)=0$ and $\left.m_{1}(\mu) \neq 0\right)$ that the coefficient of $p_{\mu}$ in $p_{1} Q_{n-1}$ is $\frac{m_{1}(\mu) 2^{l(\mu)-1}}{z_{\mu}}$. Thus the coefficient of $p_{\mu}$ in $Q_{(n-1,1)}$ is $\frac{\left(m_{1}(\mu)-2\right) 2^{l(\mu)}}{z_{\mu}}$, which by the first paragraph proves the result.

5.2. Markov chains on strict partitions. This subsection discusses a Markov chain on $D P(n)$ to be used in defining an exchangeable pair for the proof of Theorem 5.2 .

Motivated by constructions in the group and Gelfand pair cases, it would be natural, for $\tau$ a fixed element of $D P(n)$, to define a "Markov chain" $J_{\tau}$ on $D P(n)$ with transition "probabilities"

$$
J_{\tau}(\lambda, \rho):=\frac{g_{\rho}}{2^{l(\rho)} g_{\lambda} g_{\tau}} \sum_{\nu \in O P(n)} \frac{2^{l(\nu)} X_{\nu}^{\lambda} X_{\nu}^{\rho} X_{\nu}^{\tau}}{z_{\nu}} .
$$

Using Lemma 5.4, one can see that $\sum_{\rho} J_{\tau}(\lambda, \rho)=1$ for all $\lambda$. Moreover $J_{\tau}$ satisfies the reversibility condition

$$
\frac{2^{n-l(\lambda)} g_{\lambda}^{2}}{n !} J_{\tau}(\lambda, \rho)=\frac{2^{n-l(\rho)} g_{\rho}^{2}}{n !} J_{\tau}(\rho, \lambda)
$$

for all $\lambda, \rho \in D P(n)$. Unfortunately, the quantity $J_{\tau}(\lambda, \rho)$ can be negative. For instance one can check from Lemma 5.6 that $J_{(2,1)}((2,1),(2,1))=-1$.

To deal with the complication raised in the previous paragraph, it is helpful to introduce a genuine Markov chain $L$ on $D P(n)$, with transition probabilities

$$
L(\lambda, \rho)=\frac{2 g_{\rho}}{n g_{\lambda}} \sum_{\substack{\eta \in D P(n-1) \\ \eta>\lambda, \eta>\rho}} 2^{l(\eta)-l(\rho)} .
$$

Here $\eta \nearrow \lambda$ means that $\eta$ is obtained from $\lambda$ by decreasing the size of some part by exactly one.

Lemma 5.7 proves that $L$ is a Markov chain which is reversible with respect to shifted Plancherel measure. As is mentioned in the proof, the definition of $L$ was motivated by the theory of harmonic functions on Bratelli diagrams. 
Lemma 5.7. The transition probabilities of $L$ are real and non-negative and sum to 1. Moreover the chain $L$ is reversible with respect to shifted Plancherel measure.

Proof. This is a special case of a construction in Section 2 of 14. To see this, one takes the underlying Bratelli diagram to be the Schur graph, the properties of which are discussed in Section 5 of [5. The vertices of the Schur graph are all partitions of all non-negative integers with distinct parts, and the edge multiplicity between $\eta \in D P(n-1)$ and $\lambda \in D P(n)$ is 1 if $\eta \nearrow \lambda$, and is 0 otherwise. The combinatorial dimension of a shape $\lambda$ is $g_{\lambda}$, and if $\pi$ denotes shifted Plancherel measure, the function $\frac{\pi(\lambda)}{g_{\lambda}}$ is harmonic on the Schur graph.

Proposition 5.9 will establish a fundamental relation between the Markov chain $L$ and the "Markov chain" $J_{(n-1,1)}$. The argument involves symmetric function theory, and first a lemma is needed about properties of a certain subring $\Gamma$ of the ring of symmetric functions, defined on page 252 of 29 .

Recall from page 255 of 29 that there is an inner product on $\Gamma$ which satisfies the properties

(1) $\left\langle p_{\lambda}, p_{\mu}\right\rangle=2^{-l(\lambda)} z_{\lambda} \delta_{\lambda, \mu}$ if $\lambda, \mu \in O P(n)$.

(2) $\left\langle P_{\lambda}, P_{\mu}\right\rangle=2^{-l(\lambda)} \delta_{\lambda, \mu}$ if $\lambda, \mu \in D P(n)$.

Here $p_{\lambda}$ is a power sum symmetric function and $P_{\lambda}$ is a Hall-Littlewood polynomial with the parameter $t=-1$. It is also helpful to recall, from page 247 of [29], that for $\lambda \in D P(n)$,

$$
P_{\lambda}=2^{-l(\lambda)} \sum_{\nu \in O P(n)} \frac{2^{l(\nu)}}{z_{\nu}} X_{\nu}^{\lambda} p_{\nu} .
$$

Lemma 5.8. Let $p_{1}^{\perp}$ be the adjoint in $\Gamma$ of multiplication by $p_{1}$.

(1) (29], page 265) $p_{1}^{\perp}=\frac{1}{2} \frac{\partial}{\partial p_{1}}$.

(2) $p_{1}^{\perp} P_{\lambda}=\sum_{\substack{\eta \in D P(n-1) \\ \eta>\lambda}} 2^{l(\eta)-l(\lambda)} P_{\eta}$.

Proof. Only the second assertion needs to be proved. Consider the coefficient of $P_{\eta}$ in $p_{1}^{\perp} P_{\lambda}$. It is

$$
\frac{\left\langle p_{1}^{\perp} P_{\lambda}, P_{\eta}\right\rangle}{\left\langle P_{\eta}, P_{\eta}\right\rangle}=\frac{\left\langle P_{\lambda}, p_{1} P_{\eta}\right\rangle}{\left\langle P_{\eta}, P_{\eta}\right\rangle}=2^{l(\eta)}\left\langle P_{\lambda}, p_{1} P_{\eta}\right\rangle .
$$

From Section 3.8 of [29],

$$
p_{1} P_{\eta}=\sum_{\substack{\lambda \in D P(n) \\ \eta / \lambda}} P_{\lambda}
$$

which implies the result.

Proposition 5.9 can now be proved.

Proposition 5.9. Suppose that $n \geq 3$, so that $(n-1,1)$ is a strict partition. Then

$$
L(\lambda, \rho)=\frac{n-2}{n} J_{(n-1,1)}(\lambda, \rho)
$$

for $\lambda, \rho \in D P(n)$ such that $\lambda \neq \rho$. 
Proof. By Lemma 5.6 .

$$
J_{(n-1,1)}(\lambda, \rho)=\frac{g_{\rho}}{2^{l(\rho)} g_{\lambda}(n-2)} \sum_{\nu \in O P(n)} \frac{2^{l(\nu)} X_{\nu}^{\lambda} X_{\nu}^{\rho}\left(m_{1}(\nu)-2\right)}{z_{\nu}} .
$$

Since $\lambda \neq \rho$, Lemma 5.3 and part 1 of Lemma 5.8 imply that this is equal to

$$
\begin{aligned}
& \frac{g_{\rho}}{2^{l(\rho)} g_{\lambda}(n-2)} \sum_{\nu \in O P(n)} \frac{2^{l(\nu)} X_{\nu}^{\lambda} X_{\nu}^{\rho} m_{1}(\nu)}{z_{\nu}} \\
= & \frac{g_{\rho}}{2^{l(\rho)} g_{\lambda}(n-2)}\left\langle\sum_{\nu \in O P(n)} m_{1}(\nu) \frac{2^{l(\nu)}}{z_{\nu}} X_{\nu}^{\lambda} p_{\nu}, \sum_{\nu \in O P(n)} \frac{2^{l(\nu)}}{z_{\nu}} X_{\nu}^{\rho} p_{\nu}\right\rangle \\
= & \frac{2^{l(\lambda)} g_{\rho}}{g_{\lambda}(n-2)}\left\langle 2 p_{1} p_{1}^{\perp} P_{\lambda}, P_{\rho}\right\rangle \\
= & \frac{2^{l(\lambda)+1} g_{\rho}}{g_{\lambda}(n-2)}\left\langle p_{1}^{\perp} P_{\lambda}, p_{1}^{\perp} P_{\rho}\right\rangle .
\end{aligned}
$$

By part (2) of Lemma 5.8 , this is

$$
\begin{aligned}
& \frac{2^{l(\lambda)+1} g_{\rho}}{g_{\lambda}(n-2)}\left\langle\sum_{\substack{\eta \in D P(n-1) \\
\eta>\lambda}} 2^{l(\eta)-l(\lambda)} P_{\eta}, \sum_{\substack{\eta \in D P(n-1) \\
\eta>\rho}} 2^{l(\eta)-l(\rho)} P_{\eta}\right\rangle \\
= & \frac{2 g_{\rho}}{g_{\lambda}(n-2)} \sum_{\substack{\eta \in D P(n-1) \\
\eta>\lambda, \eta / \rho}} 2^{l(\eta)-l(\rho)} \\
= & \frac{n}{n-2} L(\lambda, \rho) .
\end{aligned}
$$

5.3. Central limit theorem for shifted Plancherel measure. This subsection studies the statistic $W=\sqrt{\frac{n !}{z_{\mu} 2^{n-l(\mu)}}} \frac{X_{\mu}^{\lambda}}{g_{\lambda}}$, where $\mu \in O P(n)$ is fixed and $\lambda \in D P(n)$ is chosen from shifted Plancherel measure. The main goal is a proof of Theorem 5.2. Note that Lemma 5.4 implies that $\mathbb{E}(W)=0$ if $\mu \neq\left(1^{n}\right)$ and that $\mathbb{E}\left(W^{2}\right)=1$.

Using $L$, one constructs an exchangeable pair $\left(W, W^{\prime}\right)$ as follows. Choose $\lambda$ from the shifted Plancherel measure on partitions of size $n$. Then choose $\rho$ with probability $L(\lambda, \rho)$ and let $\left(W, W^{\prime}\right)=(W(\lambda), W(\rho))$. Using $J_{\tau}$, one constructs an "exchangeable pair" $\left(W, W^{*}\right)$ as follows. Choose $\lambda$ from the shifted Plancherel measure on partitions of size $n$. Then choose $\rho$ with "probability" $J_{\tau}(\lambda, \rho)$ and let $\left(W, W^{*}\right)=(W(\lambda), W(\rho))$. The pair $\left(W, W^{\prime}\right)$ is a valid candidate for Stein's method. However the pair $\left(W, W^{*}\right)$ is much easier to work with, and by Proposition 5.9, when $\tau=(n-1,1)$, this gives insight into the genuine exchangeable pair $\left(W, W^{\prime}\right)$. Even though the transition probabilities of $J_{\tau}$ can be negative, for convenience the usual language of probability theory (expected value, variance, etc.) will be used when working with them.

Lemma 5.10. (1) $\mathbb{E}\left(W^{*} \mid W\right)=\left(\frac{X_{\mu}^{\tau}}{g_{\tau}}\right) W$.

(2) $\mathbb{E}\left(W^{\prime} \mid W\right)=\frac{m_{1}(\mu)}{n} W$. 
Proof. For the first assertion, the definition of $J_{(n-1,1)}$ and Lemma 5.4 imply that

$$
\begin{aligned}
\mathbb{E}\left(W^{*} \mid \lambda\right) & =\sqrt{\frac{n !}{z_{\mu} 2^{n-l(\mu)}}} \sum_{\rho \in D P(n)} \frac{g_{\rho}}{2^{l(\rho)} g_{\lambda} g_{\tau}} \sum_{\nu \in O P(n)} \frac{2^{l(\nu)} X_{\nu}^{\lambda} X_{\nu}^{\rho} X_{\nu}^{\tau}}{z_{\nu}} \frac{X_{\mu}^{\rho}}{g_{\rho}} \\
& =\sqrt{\frac{n !}{z_{\mu} 2^{n-l(\mu)}}} \frac{1}{g_{\lambda} g_{\tau}} \sum_{\nu \in O P(n)} \frac{2^{l(\nu)} X_{\nu}^{\lambda} X_{\nu}^{\tau}}{z_{\nu}} \sum_{\rho \in D P(n)} \frac{X_{\nu}^{\rho} X_{\mu}^{\rho}}{2^{l(\rho)}} \\
& =\sqrt{\frac{n !}{z_{\mu} 2^{n-l(\mu)}}} \frac{X_{\mu}^{\lambda}}{g_{\lambda}} \frac{X_{\mu}^{\tau}}{g_{\tau}} \\
& =\left(\frac{X_{\mu}^{\tau}}{g_{\tau}}\right) W(\lambda) .
\end{aligned}
$$

The first assertion follows since this depends on $\lambda$ only through $W$.

For the second assertion, observe that by Proposition 5.9.

$$
\begin{aligned}
\mathbb{E}\left(W^{\prime}-W \mid \lambda\right) & =\sum_{\rho} L(\lambda, \rho)(W(\rho)-W(\lambda)) \\
& =\frac{n-2}{n} \sum_{\rho} J_{(n-1,1)}(\lambda, \rho)(W(\rho)-W(\lambda)) \\
& =-\left(\frac{n-2}{n}\right) W(\lambda)+\frac{n-2}{n} \sum_{\rho} J_{(n-1,1)}(\lambda, \rho) W(\rho) .
\end{aligned}
$$

By the first assertion and Lemma [5.6, this is

$$
-\left(\frac{n-2}{n}\right) W(\lambda)+\frac{n-2}{n} \frac{m_{1}(\mu)-2}{n-2} W(\lambda)=\left(\frac{m_{1}(\mu)}{n}-1\right) W(\lambda),
$$

which implies the result.

Corollary 5.11 will not be needed but is worth recording.

Corollary 5.11. The eigenvalues of $J_{\tau}$ are $\frac{X_{\mu}^{\tau}}{g_{\tau}}$ as $\mu$ ranges over $\operatorname{OP}(n)$. The functions $\psi_{\mu}(\lambda)=\sqrt{\frac{n !}{z_{\mu} 2^{n-l(\mu)}}} \frac{X_{\mu}^{\lambda}}{g_{\lambda}}$ are a basis of eigenvectors of $J_{\tau}$, orthonormal with respect to the inner product

$$
\left\langle f_{1}, f_{2}\right\rangle=\sum_{\lambda \in D P(n)} f_{1}(\lambda) \overline{f_{2}(\lambda)} \frac{2^{n-l(\lambda)} g_{\lambda}^{2}}{n !} .
$$

Proof. The proof of part (1) of Lemma 5.10 shows that $\psi_{\mu}$ is an eigenvector of $J_{\tau}$ with eigenvalue $\frac{X_{\mu}^{\tau}}{g_{\tau}}$. The orthonormality assertion follows from Lemma 5.4 and the fact from [29] that all $X_{\mu}^{\lambda}$ are real valued. The basis assertion follows since $|D P(n)|=|O P(n)|$.

Lemma 5.12. $\mathbb{E}\left(W^{*}-W\right)^{2}=2\left(1-\frac{X_{\mu}^{\tau}}{g_{\tau}}\right)$.

Proof. This follows from the proof of Lemma 2.3 (which does not require nonnegative transition probabilities) and part (1) of Lemma 5.10 
For the remainder of this subsection, $p_{m}\left(\tilde{K}_{\nu}\right)$ will denote the coefficient of $\tilde{K}_{\nu}$ in $\left(\tilde{K}_{\mu}\right)^{m}$. When $m=0$ this is to be interpreted through Lemma 5.5, so that $p_{0}\left(\tilde{K}_{\left(1^{n}\right)}\right)=1$ and $p_{0}\left(\tilde{K}_{\nu}\right)=0$ for $\nu \neq\left(1^{n}\right)$. Due to the signs in the definition of $\tilde{K}_{\nu}$, these numbers are not probabilities, so care must be taken in working with them. For instance it is not true that $\sum_{\nu \in O P(n)} p_{m}\left(\tilde{K}_{\nu}\right)=1$. However the following three relations will be useful.

Lemma 5.13. $\sum_{\nu \in O P(n)} p_{2}\left(\tilde{K}_{\nu}\right) 2^{l(\nu)-n}=2^{2[l(\mu)-n]}$.

Proof. Using Lemma [5.5, one has that

$$
\sum_{\nu \in O P(n)} p_{2}\left(\tilde{K}_{\nu}\right) 2^{l(\nu)}=2^{2 l(\mu)-n} \sum_{\rho \in D P(n)} 2^{-l(\rho)} \frac{\left(X_{\mu}^{\rho}\right)^{2}}{g_{\rho}} \sum_{\nu \in O P(n)} \frac{2^{l(\nu)}}{z_{\nu}} X_{\nu}^{\rho} .
$$

By page 248 of $\left[29, X_{\nu}^{n}=1\right.$, so that Lemma 5.3 implies that

$$
\sum_{\nu \in O P(n)} \frac{2^{l(\nu)}}{z_{\nu}} X_{\nu}^{\rho} X_{\nu}^{n}=2 \delta_{\rho, n}
$$

The result follows.

Lemma 5.14. $\mathbb{E}\left(\frac{X_{\mu}^{\lambda}}{g_{\lambda}}\right)^{4}=\left(2^{n-l(\mu)}\right)^{4} \sum_{\nu \in O P(n)} p_{2}\left(\tilde{K}_{\nu}\right)^{2} \frac{2^{l(\nu)} z_{\nu}}{2^{n} n !}$.

Proof. Consider the coefficient of $\tilde{K}_{\left(1^{n}\right)}$ in $\left(\tilde{K}_{\mu}\right)^{4}$. On one hand, by Lemma [5.5] it is equal to $\left(2^{l(\mu)-n}\right)^{4} \mathbb{E}\left(\frac{X_{\mu}^{\lambda}}{g_{\lambda}}\right)^{4}$. On the other hand,

$$
\left(\tilde{K}_{\mu}\right)^{4}=\left(\tilde{K}_{\mu}\right)^{2}\left(\tilde{K}_{\mu}\right)^{2}=\left[\sum_{\nu \in O P(n)} p_{2}\left(\tilde{K}_{\nu}\right) \tilde{K}_{\nu}\right]^{2} .
$$

From Lemmas 5.5 and Lemma 5.4 it follows that the coefficient of $\tilde{K}_{\left(1^{n}\right)}$ in $\tilde{K}_{\nu_{1}} \tilde{K}_{\nu_{2}}$ is 0 if $\nu_{1} \neq \nu_{2}$, and that the coefficient of $\tilde{K}_{\left(1^{n}\right)}$ in $\left(\tilde{K}_{\nu}\right)^{2}$ is $\frac{2^{l(\nu)-n} z_{\nu}}{n !}$. Thus the coefficient of $\tilde{K}_{\left(1^{n}\right)}$ in $\left(\tilde{K}_{\mu}\right)^{4}$ is equal to

$$
\sum_{\nu \in O P(n)} p_{2}\left(\tilde{K}_{\nu}\right)^{2} \frac{2^{l(\nu)-n} z_{\nu}}{n !} .
$$

Comparing the two expressions for the coefficient of $\tilde{K}_{\left(1^{n}\right)}$ in $\left(\tilde{K}_{\mu}\right)^{4}$ proves the result.

Lemma 5.15. $p_{3}\left(\tilde{K}_{\mu}\right)=\frac{2^{n-l(\mu)}}{z_{\mu}} \sum_{\nu \in O P(n)} p_{2}\left(\tilde{K}_{\nu}\right)^{2} 2^{l(\nu)-n} z_{\nu}$.

Proof. By Lemma 5.5,

$$
p_{3}\left(\tilde{K}_{\mu}\right)=\frac{n !\left(2^{l(\mu)-n}\right)^{3}}{z_{\mu}} \mathbb{E}\left(\frac{X_{\mu}^{\lambda}}{g_{\lambda}}\right)^{4} .
$$

Now use Lemma 5.14

The next lemmas are crucial. 
Lemma 5.16.

(1)

$$
\mathbb{E}\left[\left(W^{*}-W\right)^{2} \mid \lambda\right]=\frac{n !}{z_{\mu} 2^{l(\mu)}} \sum_{\nu \in O P(n)} 2^{l(\nu)} p_{2}\left(\tilde{K}_{\nu}\right)\left(\frac{X_{\nu}^{\tau}}{g_{\tau}}+1-\frac{2 X_{\mu}^{\tau}}{g_{\tau}}\right) \frac{X_{\nu}^{\lambda}}{g_{\lambda}} .
$$

$$
\operatorname{Var}\left(\mathbb{E}\left[\left(W^{*}-W\right)^{2} \mid \lambda\right]\right)=\frac{n ! 2^{n}}{z_{\mu}^{2} 2^{2 l(\mu)}} \sum_{\substack{\nu \in O P(n) \\ \nu \neq(1 n)}} 2^{l(\nu)} p_{2}\left(\tilde{K}_{\nu}\right)^{2} z_{\nu}\left(\frac{X_{\nu}^{\tau}}{g_{\tau}}+1-\frac{2 X_{\mu}^{\tau}}{g_{\tau}}\right)^{2} .
$$

Proof. Note that $\mathbb{E}\left(\left(W^{*}\right)^{2} \mid \lambda\right)$ is equal to

$$
\begin{aligned}
& \frac{n !}{z_{\mu} 2^{n-l(\mu)}} \sum_{\rho \in D P(n)} \frac{g_{\rho}}{2^{l(\rho)} g_{\lambda} g_{\tau}} \sum_{\nu \in O P(n)} \frac{2^{l(\nu)} X_{\nu}^{\lambda} X_{\nu}^{\rho} X_{\nu}^{\tau}}{z_{\nu}}\left(\frac{X_{\mu}^{\rho}}{g_{\rho}}\right)^{2} \\
= & \frac{n !}{z_{\mu} 2^{n-l(\mu)}} \sum_{\nu \in O P(n)} \frac{X_{\nu}^{\lambda}}{g_{\lambda}} \frac{X_{\nu}^{\tau}}{g_{\tau}} 2^{l(\nu)}\left(\frac{1}{z_{\nu}} \sum_{\rho \in D P(n)} \frac{g_{\rho}^{2}}{2^{l(\rho)}} \frac{X_{\nu}^{\rho}}{g_{\rho}}\left(\frac{X_{\mu}^{\rho}}{g_{\rho}}\right)^{2}\right) \\
= & \frac{n !}{z_{\mu} 2^{l(\mu)}} \sum_{\nu \in O P(n)} \frac{X_{\nu}^{\lambda}}{g_{\lambda}} \frac{X_{\nu}^{\tau}}{g_{\tau}} 2^{l(\nu)} p_{2}\left(\tilde{K}_{\nu}\right) .
\end{aligned}
$$

The first equality switched the order of summation and the second equality is Lemma 5.5

Next we claim that $W^{2}=\frac{n !}{z_{\mu} 2^{l(\mu)}} \sum_{\nu \in O P(n)} 2^{l(\nu)} p_{2}\left(\tilde{K}_{\nu}\right) \frac{X_{\nu}^{\lambda}}{g_{\lambda}}$. To see this note by Lemma 5.5 that

$$
\frac{2^{2 l(\mu)-2 n}}{z_{\nu}} \sum_{\rho \in D P(n)} 2^{n-l(\rho)} \frac{X_{\nu}^{\rho}}{g_{\rho}}\left(X_{\mu}^{\rho}\right)^{2}=p_{2}\left(\tilde{K}_{\nu}\right)
$$

Multiplying both sides by $\frac{2^{l(\nu)} n !}{2^{l(\mu)} z_{\mu}} \frac{X_{\nu}^{\lambda}}{g_{\lambda}}$, and summing over $\nu \in O P(n)$, the claimed expression for $W^{2}$ follows from Lemma 5.3 .

Now observe from Lemma 5.10 that

$$
\begin{aligned}
\mathbb{E}\left[\left(W^{*}-W\right)^{2} \mid \lambda\right] & =\mathbb{E}\left(\left(W^{*}\right)^{2} \mid \lambda\right)-2 W \mathbb{E}\left(W^{*} \mid \lambda\right)+W^{2} \\
& =\mathbb{E}\left(\left(W^{*}\right)^{2} \mid \lambda\right)+\left(1-\frac{2 X_{\mu}^{\tau}}{g_{\tau}}\right) W^{2} .
\end{aligned}
$$

The first part of the lemma follows from this and the previous two paragraphs.

From the first part of the lemma and Lemma 5.4, it follows that

$$
\mathbb{E}\left(\mathbb{E}\left[\left(W^{*}-W\right)^{2} \mid \lambda\right]^{2}\right)=\frac{n ! 2^{n}}{z_{\mu}^{2} 2^{2 l(\mu)}} \sum_{\nu \in O P(n)} 2^{l(\nu)} p_{2}\left(\tilde{K}_{\nu}\right)^{2} z_{\nu}\left(\frac{X_{\nu}^{\tau}}{g_{\tau}}+1-\frac{2 X_{\mu}^{\tau}}{g_{\tau}}\right)^{2} .
$$

Lemmas 5.5 and 5.4 imply that $p_{2}\left(\tilde{K}_{\left(1^{n}\right)}\right)=\frac{z_{\mu}}{n ! 2^{n-l(\mu)}}$. The second part of the lemma now follows since by Lemma 5.12 .

$$
\operatorname{Var}\left(\mathbb{E}\left[\left(W^{*}-W\right)^{2} \mid \lambda\right]\right)=\mathbb{E}\left(\mathbb{E}\left[\left(W^{*}-W\right)^{2} \mid \lambda\right]^{2}\right)-4\left(1-\frac{X_{\mu}^{\tau}}{g_{\tau}}\right)^{2} .
$$


Lemma 5.17. Let $k$ be a positive integer.

(1) $\mathbb{E}\left(W^{*}-W\right)^{k}$ is equal to

$$
\left(\frac{n ! 2^{n-l(\mu)}}{z_{\mu}}\right)^{k / 2} \sum_{m=0}^{k}(-1)^{k-m}\left(\begin{array}{c}
k \\
m
\end{array}\right) \sum_{\nu \in O P(n)} \frac{X_{\nu}^{\tau} z_{\nu}}{g_{\tau} n ! 2^{n-l(\nu)}} p_{m}\left(\tilde{K}_{\nu}\right) p_{k-m}\left(\tilde{K}_{\nu}\right) .
$$

(2) $\mathbb{E}\left(W^{*}-W\right)^{4}$ is equal to

$$
\left(\frac{n ! 2^{n-l(\mu)}}{z_{\mu}}\right)^{2}\left[\sum_{\nu \in O P(n)}\left(8\left(1-\frac{X_{\mu}^{\tau}}{g_{\tau}}\right)-6\left(1-\frac{X_{\nu}^{\tau}}{g_{\tau}}\right)\right) \frac{z_{\nu} p_{2}\left(\tilde{K}_{\nu}\right)^{2}}{n ! 2^{n-l(\nu)}}\right] .
$$

Proof. To prove the first assertion, observe that

$$
\begin{aligned}
& \mathbb{E}\left(\left(W^{*}-W\right)^{k} \mid \lambda\right) \\
= & \left(\frac{n !}{z_{\mu} 2^{n-l(\mu)}}\right)^{k / 2} \sum_{\rho \in D P(n)} \frac{g_{\rho}}{2^{l(\rho)} g_{\lambda} g_{\tau}} \sum_{\nu \in O P(n)} \frac{2^{l(\nu)} X_{\nu}^{\lambda} X_{\nu}^{\rho} X_{\nu}^{\tau}}{z_{\nu}} \\
& \cdot \sum_{m=0}^{k}(-1)^{k-m}\left(\begin{array}{c}
k \\
m
\end{array}\right)\left(\frac{X_{\mu}^{\rho}}{g_{\rho}}\right)^{m}\left(\frac{X_{\mu}^{\lambda}}{g_{\lambda}}\right)^{k-m} \\
= & \left(\frac{n !}{z_{\mu} 2^{n-l(\mu)}}\right)^{k / 2} \frac{1}{g_{\lambda} g_{\tau}} \sum_{m=0}^{k}(-1)^{k-m}\left(\begin{array}{c}
k \\
m
\end{array}\right)\left(\frac{X_{\mu}^{\lambda}}{g_{\lambda}}\right)^{k-m} \\
& \cdot \sum_{\nu \in O P(n)} \frac{2^{l(\nu)} X_{\nu}^{\lambda} X_{\nu}^{\tau}}{z_{\nu}} \sum_{\rho \in D P(n)} \frac{g_{\rho}^{2}}{2^{l(\rho)}} \frac{X_{\nu}^{\rho}}{g_{\rho}}\left(\frac{X_{\mu}^{\rho}}{g_{\rho}}\right)^{m} \\
= & \left(\frac{n !}{z_{\mu} 2^{n-l(\mu)}}\right)^{k / 2} \frac{1}{2^{n} g_{\lambda} g_{\tau}} \sum_{m=0}^{k}(-1)^{k-m}\left(\begin{array}{c}
k \\
m
\end{array}\right)\left(2^{n-l(\mu)}\right)^{m}\left(\frac{X_{\mu}^{\lambda}}{g_{\lambda}}\right)^{k-m} \\
& \cdot \sum_{\nu \in O P(n)} 2^{l(\nu)} X_{\nu}^{\lambda} X_{\nu}^{\tau} p_{m}\left(\tilde{K}_{\nu}\right) .
\end{aligned}
$$

The second equality switched the order of summation and the last equation is Lemma 5.5. Hence $\mathbb{E}\left(W^{*}-W\right)^{k}$ is equal to

$$
\begin{aligned}
& \mathbb{E}\left(\mathbb{E}\left(\left(W^{*}-W\right)^{k} \mid \lambda\right)\right) \\
= & \left(\frac{n !}{z_{\mu} 2^{n-l(\mu)}}\right)^{k / 2} \frac{\left(2^{n-l(\mu)}\right)^{k}}{2^{n}} \sum_{m=0}^{k}(-1)^{k-m}\left(\begin{array}{c}
k \\
m
\end{array}\right) \\
& \cdot \sum_{\nu \in O P(n)} 2^{l(\nu)} \frac{X_{\nu}^{\tau}}{g_{\tau}} \frac{p_{m}\left(\tilde{K}_{\nu}\right)}{\left(2^{n-l(\mu)}\right)^{k-m}} \mathbb{E}\left[\frac{X_{\nu}^{\lambda}}{g_{\lambda}}\left(\frac{X_{\mu}^{\lambda}}{g_{\lambda}}\right)^{k-m}\right] .
\end{aligned}
$$

The first assertion now follows from Lemma 5.5 .

For the second assertion, the first assertion gives that $\mathbb{E}\left(W^{*}-W\right)^{4}$ is equal to

$$
\left(\frac{n ! 2^{n-l(\mu)}}{z_{\mu}}\right)^{2} \sum_{m=0}^{4}(-1)^{m}\left(\begin{array}{c}
4 \\
m
\end{array}\right) \sum_{\nu \in O P(n)} \frac{z_{\nu}}{2^{n-l(\nu)} n !} \frac{X_{\nu}^{\tau}}{g_{\tau}} p_{m}\left(\tilde{K}_{\nu}\right) p_{4-m}\left(\tilde{K}_{\nu}\right) .
$$


The special case $\tau=(n)$ gives that

$$
0=\left(\frac{n ! 2^{n-l(\mu)}}{z_{\mu}}\right)^{2} \sum_{m=0}^{4}(-1)^{m}\left(\begin{array}{c}
4 \\
m
\end{array}\right) \sum_{\nu \in O P(n)} \frac{z_{\nu}}{2^{n-l(\nu)} n !} p_{m}\left(\tilde{K}_{\nu}\right) p_{4-m}\left(\tilde{K}_{\nu}\right) .
$$

Thus in general, $\mathbb{E}\left(W^{*}-W\right)^{4}$ is equal to

$$
-\left(\frac{n ! 2^{n-l(\mu)}}{z_{\mu}}\right)^{2} \sum_{m=0}^{4}(-1)^{m}\left(\begin{array}{c}
4 \\
m
\end{array}\right) \sum_{\nu \in O P(n)}\left(1-\frac{X_{\nu}^{\tau}}{g_{\tau}}\right) \frac{z_{\nu} p_{m}\left(\tilde{K}_{\nu}\right) p_{4-m}\left(\tilde{K}_{\nu}\right)}{2^{n-l(\nu)} n !} .
$$

The $m=0,4$ terms vanish since only $\nu=\left(1^{n}\right)$ could contribute, but it contributes 0 . The $m=2$ term contributes

$$
-6\left(\frac{n ! 2^{n-l(\mu)}}{z_{\mu}}\right)^{2} \sum_{\nu \in O P(n)}\left(1-\frac{X_{\nu}^{\tau}}{g_{\tau}}\right) \frac{z_{\nu} p_{2}\left(\tilde{K}_{\nu}\right)^{2}}{n ! 2^{n-l(\nu)}} .
$$

The $m=1,3$ terms are equal and together contribute

$$
8\left(\frac{n ! 2^{n-l(\mu)}}{z_{\mu}}\right)\left(1-\frac{X_{\mu}^{\tau}}{g_{\tau}}\right) p_{3}\left(\tilde{K}_{\mu}\right) .
$$

By Lemma 5.15, this is

$$
8\left(\frac{n ! 2^{n-l(\mu)}}{z_{\mu}}\right)^{2}\left(1-\frac{X_{\mu}^{\tau}}{g_{\tau}}\right) \sum_{\nu \in O P(n)} \frac{z_{\nu} p_{2}\left(\tilde{K}_{\nu}\right)^{2}}{n ! 2^{n-l(\nu)}} .
$$

Adding the terms together proves the second assertion.

The final combinatorial ingredient for the proof of Theorem $[5.2$ is an upper bound for $p_{2}\left(\tilde{K}_{\nu}\right)$. Lemma 5.18 reduces this to a result obtained earlier in the section on Gelfand pairs.

Lemma 5.18. Let $p_{2}\left(\tilde{K}_{\nu}\right)$ be as in this subsection and let $p_{2}\left(K_{\nu}\right)$ be as in Subsection 4.4. Then $p_{2}\left(\tilde{K}_{\nu}\right) \leq p_{2}\left(K_{\nu}\right)$ for all $\nu \in O P(n)$.

Proof. Clearly $p_{2}\left(K_{\nu}\right)$ is equal to $\left|B_{n} \omega_{\nu} B_{n}\right|$ multiplied by the coefficient of $\omega_{\nu}$ in

$$
\left(\frac{1}{\left|B_{n}\right|^{2}} \sum_{x_{1}, x_{2} \in B_{n}} x_{1} \omega_{\mu} x_{2}\right)^{2} \text {. }
$$

Next consider $p_{2}\left(\tilde{K}_{\nu}\right)$. By definition it is the coefficient of $\tilde{K}_{\nu}$ in $\left(\tilde{K}_{\mu}\right)^{2}$. Corollary 3.2 of [42] gives that the coefficient of $\omega_{\nu}$ in $\tilde{K}_{\nu}$ is $\frac{1}{\mid B_{n} \omega_{\nu} B_{n}}$. Hence $p_{2}\left(\tilde{K}_{\nu}\right)$ is $\left|B_{n} \omega_{\nu} B_{n}\right|$ multiplied by the coefficient of $\omega_{\nu}$ in

$$
\left(\frac{1}{\left|B_{n}\right|^{2}} \sum_{x_{1}, x_{2} \in B_{n}} \phi\left(x_{1} x_{2}\right) x_{1} \omega_{\mu} x_{2}\right)^{2},
$$

where $\phi$ is the linear character of $B_{n}$ described in Subsection 4.1. Since $\phi\left(x_{1} x_{2}\right)$ is always \pm 1 , the result follows.

Finally, we prove Theorem 5.2 
Proof of Theorem [5.2. One can assume that $n \geq 2(2 i+1)$. In the $O$ notation throughout the proof, $i$ is fixed and $n$ is growing.

One applies Theorem 2.1 to the pair $\left(W, W^{\prime}\right)$, where $\mu=\left(2 i+1,1^{n-2 i-1}\right)$. Then $a=1-\frac{m_{1}(\mu)}{n}$ by part (2) of Lemma [5.10. Proposition [5.9] implies that

$$
\mathbb{E}\left(\left(W^{\prime}-W\right)^{2} \mid \lambda\right)=\frac{n-2}{n} \mathbb{E}\left(\left(W^{*}-W\right)^{2} \mid \lambda\right)
$$

for all $\lambda$. Thus

$$
\operatorname{Var}\left(\mathbb{E}\left[\left(W^{\prime}-W\right)^{2} \mid \lambda\right]\right)=\left(\frac{n-2}{n}\right)^{2} \operatorname{Var}\left(\mathbb{E}\left[\left(W^{*}-W\right)^{2} \mid \lambda\right]\right) .
$$

Also note, as in the proof of Theorem 3.12, that

$$
\operatorname{Var}\left(\mathbb{E}\left[\left(W^{\prime}-W\right)^{2} \mid W\right]\right) \leq \operatorname{Var}\left(\mathbb{E}\left[\left(W^{\prime}-W\right)^{2} \mid \lambda\right]\right) .
$$

Lemmas 5.16 and 5.6 give that the first error term in applying Theorem 2.1 to the pair $\left(W, W^{\prime}\right)$ is at most

$$
\frac{n-2}{n} \frac{n ! 2^{n-l(\mu)}}{\left(1-\frac{m_{1}(\mu)}{n}\right) z_{\mu}} \sqrt{\sum_{\substack{\nu \in O P(n) \\ \nu \neq\left(1^{n}\right)}} \frac{z_{\nu} p_{2}\left(\tilde{K}_{\nu}\right)^{2}}{n ! 2^{n-l(\nu)}}\left(\frac{n+m_{1}(\nu)-2 m_{1}(\mu)}{n-2}\right)^{2}} .
$$

Observe that $\frac{n-2}{n} \frac{n ! 2^{n-l(\mu)}}{\left(1-\frac{m_{1}(\mu)}{n}\right) z_{\mu}}$ is $O\left(n^{2 i+2}\right)$. In the sum over $\nu \neq\left(1^{n}\right)$, the term coming from $\nu=\left(2 i+1,2 i+1,1^{n-4 i-2}\right)$ contributes 0 . Since $n \geq 2(2 i+1)$, Lemmas 5.18 and 4.18 imply that $\frac{z_{\nu} p_{2}\left(\tilde{K}_{\nu}\right)^{2}}{n ! 2^{n-l(\nu)}}=O\left(n^{-4 i-3}\right)$ for all other $\nu$ in the sum, and the number of such $\nu$ is bounded by a constant depending on $i$ but not $n$. Moreover if $p_{2}\left(\widetilde{K}_{\nu}\right) \neq 0$, then $p_{2}\left(K_{\nu}\right) \neq 0$, implying by Lemma 4.17 that $m_{1}(\nu) \geq n-4 i-2$ and thus that $\frac{n+m_{1}(\nu)-2 m_{1}(\mu)}{n-2}=O\left(n^{-1}\right)$. Combining these observations gives us that the first term in the upper bound of Theorem 2.1 is $O\left(n^{-1 / 2}\right)$.

By the Cauchy-Schwarz inequality,

$$
\mathbb{E}\left|W^{\prime}-W\right|^{3} \leq \sqrt{\mathbb{E}\left(W^{\prime}-W\right)^{2} \mathbb{E}\left(W^{\prime}-W\right)^{4}} .
$$

Proposition 5.9 implies that $\mathbb{E}\left(W^{\prime}-W\right)^{k}=\frac{n-2}{n} \mathbb{E}\left(W^{*}-W\right)^{k}$ for all $k$. Hence Lemmas 5.12 and 5.6 imply that the second error term in Theorem 2.1 is at most

$$
\left[\frac{(n-2)}{\pi n} \frac{\mathbb{E}\left(W^{*}-W\right)^{4}}{\left(1-\frac{m_{1}(\mu)}{n}\right)}\right]^{1 / 4} .
$$

By part (2) of Lemma 5.17 and Lemma 5.6, this can be written as

$$
\left[\frac{1}{\pi} \frac{\left(n ! 2^{n-l(\mu)}\right)^{2}}{\left(1-\frac{m_{1}(\mu)}{n}\right)\left(z_{\mu}\right)^{2}} \sum_{\nu \in O P(n)}\left(2-\frac{8 m_{1}(\mu)}{n}+\frac{6 m_{1}(\nu)}{n}\right) \frac{z_{\nu} p_{2}\left(\tilde{K}_{\nu}\right)^{2}}{n ! 2^{n-l(\nu)}}\right]^{1 / 4} .
$$

The quantity $\frac{\left(n ! 2^{n-l(\mu)}\right)^{2}}{\left(1-\frac{m_{1}(\mu)}{n}\right)\left(z_{\mu}\right)^{2}}$ is $O\left(n^{4 i+3}\right)$. The contribution from $\nu=\left(1^{n}\right)$ to the sum is $\frac{8(2 i+1)}{n} p_{2}\left(\tilde{K}_{\left(1^{n}\right)}\right)^{2}$, which by Lemmas 5.5 and 5.4 is $\frac{8(2 i+1)^{3}}{2^{4 i}} n^{-4 i-3}+O\left(n^{-4 i-4}\right)$. By Lemmas 5.18 and 4.18 the contribution to the sum from $\nu$ not equal to either $\left(1^{n}\right)$ or $\left(2 i+1,2 i+1,1^{n-4 i-2}\right)$ is $O\left(n^{-4 i-4}\right)$, and the number of such $\nu$ 
with $p_{2}\left(\tilde{K}_{\nu}\right) \neq 0$ is bounded by a constant depending on $i$. The contribution to the sum from $\nu=\left(2 i+1,2 i+1,1^{n-4 i-2}\right)$ is $-\frac{4(2 i+1)}{n} \frac{z_{\nu} p_{2}\left(\tilde{K}_{\nu}\right)^{2}}{n ! 2^{n-l(\nu)}}$. Lemma 5.13 implies that $p_{2}\left(\tilde{K}_{\left(2 i+1,2 i+1,1^{n-4 i-2}\right)}\right)=1+O\left(n^{-1}\right)$, since $p_{2}\left(\tilde{K}_{\nu}\right)=O\left(n^{-1}\right)$ for $\nu \neq\left(2 i+1,2 i+1,1^{n-4 i-2}\right)$ by Lemma 5.18. Thus the contribution from $\nu=$ $\left(2 i+1,2 i+1,1^{n-4 i-2}\right)$ is $-\frac{8(2 i+1)^{3}}{2^{4 i}} n^{-4 i-3}+O\left(n^{-4 i-4}\right)$. Hence there is useful cancellation and

$$
\sum_{\nu \in O P(n)}\left(2-\frac{8 m_{1}(\mu)}{n}+\frac{6 m_{1}(\nu)}{n}\right) \frac{z_{\nu} p_{2}\left(\tilde{K}_{\nu}\right)^{2}}{n ! 2^{n-l(\nu)}}=O\left(n^{-4 i-4}\right) .
$$

It follows that the second term in the upper bound of Theorem 2.1 is $O\left(n^{-1 / 4}\right)$, completing the proof.

\section{Association schemes}

This final section adapts techniques from earlier sections to study the spectrum of an adjacency matrix of an association scheme. As is well known (see for instance Chapter 3 of [2]) this includes the spectrum of a distance regular graph as a special case.

Subsection 6.1 discusses needed facts about association schemes. Subsection 6.2 derives a general central limit theorem for the spectrum of an association scheme. Subsection 6.3 illustrates the theory of Subsection 6.2 for the special case of the Hamming scheme, where the result amounts to a central limit theorem for the spectrum of the Hamming graph, or equivalently for values of $q$-Krawtchouk polynomials.

6.1. Background on association schemes. This subsection gives preliminaries about association schemes, using notation from [30. Another useful reference is [2], and what we call association schemes some authors call symmetric association schemes.

Definition. An association scheme with $n$ classes consists of a finite set $X$ with $n+1$ relations $R_{0}, \cdots, R_{n}$ defined on $X$ which satisfy:

(1) Each $R_{i}$ is symmetric: $(x, y) \in R_{i} \Rightarrow(y, x) \in R_{i}$.

(2) For every $x, y \in X$, one has that $(x, y) \in R_{i}$ for exactly one $i$.

(3) $R_{0}=\{(x, x): x \in X\}$ is the identity relation.

(4) If $(x, y) \in R_{k}$, the number of $z \in X$ such that $(x, z) \in R_{i}$ and $(y, z) \in R_{j}$ is a constant $c_{i j k}$ depending on $i, j, k$ but not on the particular choice of $x$ and $y$.

The adjacency matrix $D_{i}$ corresponding to the relation $R_{i}$ is the $|X| \times|X|$ matrix with rows and columns labeled by the points of $X$ defined by

$$
\left(D_{i}\right)_{x, y}= \begin{cases}1 & \text { if }(x, y) \in R_{i} \\ 0 & \text { otherwise }\end{cases}
$$

The Bose-Melner algebra is defined to be the vector space consisting of all matrices $\sum_{i=0}^{n} a_{i} D_{i}$ with $a_{i}$ real. Since the matrices in the Bose-Melner algebra are symmetric and commute with each other (by parts (1) and (4) of the definition of an association scheme), the Bose-Melner algebra is semisimple and has a distinguished 
basis of primitive idempotents $J_{0}, \cdots, J_{n}$ satisfying

(1) $J_{i}^{2}=J_{i}, \quad i=0, \cdots, n$.

(2) $J_{i} J_{k}=0, \quad i \neq k$.

(3) $\sum_{i=0}^{n} J_{i}=I$.

Here $I$ is the identity matrix.

The $D$ 's are also a basis of the Bose-Melner algebra, so one can write

$$
D_{s}=\sum_{i=0}^{n} \phi_{s}(i) J_{i}, \quad s=0, \cdots, n
$$

where the $\phi_{s}(i)$ are real numbers. Since $D_{s} J_{i}=\phi_{s}(i) J_{i}$, the $\phi_{s}(i)$ are the eigenvalues of $D_{s}$. For later use note that since $D_{0}$ and $\sum_{i=0}^{n} J_{i}$ are both the identity matrix, $\phi_{0}(j)=1$ for all $j$.

Let $\mu_{i}$ be the rank of $J_{i}$. For all $s$, this is the multiplicity of the eigenvalue $\phi_{s}(i)$ of $D_{s}$. We define the Plancherel measure of the association scheme to be the probability measure on $\{0, \cdots, n\}$ which chooses $i$ with probability $\frac{\mu_{i}}{|X|}$.

Lemmas 6.1 and 6.2 are the orthogonality relations for eigenvalues of association schemes. To state them it is helpful to define $v_{i}=c_{i i 0}$, so that for any $x$, one has that $v_{i}$ is the number of pairs $(x, y) \in R_{i}$. Clearly $\sum_{i=0}^{n} v_{i}=|X|$.

Lemma 6.1 ([30], page 655). For $0 \leq k, l \leq n$,

$$
\sum_{r=0}^{n} \frac{\phi_{r}(k) \phi_{r}(l)}{v_{r}}=\frac{|X|}{\mu_{k}} \delta_{k, l}
$$

Lemma 6.2 ([30], page 655). For $0 \leq k, l \leq n$,

$$
\sum_{i=0}^{n} \mu_{i} \phi_{k}(i) \phi_{l}(i)=|X| v_{k} \delta_{k, l}
$$

Lemma 6.3 will be crucial.

Lemma 6.3. The coefficient of $D_{l}$ in $D_{s_{1}} \cdots D_{s_{m}}$ is equal to

$$
\frac{1}{v_{l}} \sum_{i=0}^{n} \frac{\mu_{i}}{|X|} \phi_{s_{1}}(i) \cdots \phi_{s_{m}}(i) \phi_{l}(i) .
$$

In particular, the coefficient of $D_{l}$ in $\left(D_{s}\right)^{m}$ is

$$
\frac{1}{v_{l}} \mathbb{E}\left[\phi_{s}(i)^{m} \phi_{l}(i)\right]
$$

where $i$ is random from the Plancherel measure of the association scheme.

Proof. Since $D_{s}=\sum_{i=0}^{n} \phi_{s}(i) J_{i}$, one knows that

$$
D_{s_{1}} \cdots D_{s_{m}}=\sum_{i=0}^{n} \phi_{s_{1}}(i) \cdots \phi_{s_{m}}(i) J_{i} .
$$

By pages 654 and 655 of [30],

$$
J_{i}=\frac{1}{|X|} \sum_{l=0}^{n} \frac{\mu_{i} \phi_{l}(i)}{v_{l}} D_{l} .
$$

The result follows. 
Let $p_{m}(r)$ denote $\frac{v_{r}}{\left(v_{s}\right)^{m}}$ multiplied by the coefficient of $D_{r}$ in $\left(D_{s}\right)^{m}$. It follows from the definitions that $p_{m}(r)$ admits the following probabilistic interpretation. Start from some point $x_{0} \in X$, move to a random $x_{1} \in X$ such that $\left(x_{0}, x_{1}\right) \in R_{s}$, then to a random $x_{2} \in X$ such that $\left(x_{1}, x_{2}\right) \in R_{s}$, and so on until one obtains $x_{m}$. Then $p_{m}(r)$ is the probability that $\left(x_{0}, x_{m}\right) \in R_{r}$.

The following fact will be useful.

Lemma 6.4. (1) $p_{4}(0)=\sum_{r=0}^{n} \frac{p_{2}(r)^{2}}{v_{r}}$.

(2) $p_{3}(s)=v_{s} \sum_{r=0}^{n} \frac{p_{2}(r)^{2}}{v_{r}}$.

Proof. The first assertion is clear from the probabilistic interpretation of $p_{m}(r)$. For an analytic proof, note that by Lemma 6.3 (in the first and fourth equalities) and Lemma 6.1 (in the second and third equalities)

$$
\begin{aligned}
\sum_{r=0}^{n} \frac{p_{2}(r)^{2}}{v_{r}} & =\sum_{r=0}^{n} \frac{1}{v_{r}}\left(\frac{1}{\left(v_{s}\right)^{2}} \sum_{i=0}^{n} \frac{\mu_{i}}{|X|} \phi_{s}(i)^{2} \phi_{r}(i)\right)^{2} \\
& =\frac{1}{\left(v_{s}\right)^{4}} \sum_{r=0}^{n} \frac{1}{v_{r}} \sum_{i=0}^{n} \frac{\left(\mu_{i}\right)^{2}}{|X|^{2}} \phi_{s}(i)^{4} \phi_{r}(i)^{2} \\
& =\frac{1}{\left(v_{s}\right)^{4}} \sum_{i=0}^{n} \frac{\mu_{i}}{|X|} \phi_{s}(i)^{4} \\
& =p_{4}(0) .
\end{aligned}
$$

The second assertion follows from the first assertion since $p_{3}(s)=v_{s} p_{4}(0)$, as can be seen either by the probabilistic interpretation of $p_{m}(r)$ or by computing both sides of the equation using Lemma 6.3.

6.2. Central limit theorem for the spectrum. Recall that the goal is to study Plancherel measure of association schemes, which chooses $i \in\{0, \cdots, n\}$ with probability $\frac{\mu_{i}}{|X|}$. More precisely, for $s$ fixed, a central limit theorem is proved for the random variable $W$ whose value at $i \in\{0, \cdots, n\}$ is $\frac{\phi_{s}(i)}{\sqrt{v_{s}}}$. Note by Lemma 6.2 that $\mathbb{E}(W)=0$ if $s \neq 0$ and that $\mathbb{E}\left(W^{2}\right)=1$.

Given $t \in\{0, \cdots, n\}$, we define a Markov chain $L_{t}$ on the set $\{0, \cdots, n\}$ which moves from $i$ to $j$ with probability

$$
L_{t}(i, j):=\frac{\mu_{j}}{|X|} \sum_{r=0}^{n} \frac{\phi_{r}(i) \phi_{r}(t) \phi_{r}(j)}{\left(v_{r}\right)^{2}} .
$$

Lemma 6.5. The transition probabilities of $L_{t}$ are real and non-negative and sum to 1. Moreover the chain $L_{t}$ is reversible with respect to Plancherel measure.

Proof. By Theorems 3.6 and 3.8 of [2], the $L_{t}(i, j)$ are non-negative real numbers. Next, observe that

$$
\sum_{j=0}^{n} L_{t}(i, j)=\frac{1}{|X|} \sum_{r=0}^{n} \frac{\phi_{r}(i) \phi_{r}(t)}{\left(v_{r}\right)^{2}} \sum_{j=0}^{n} \mu_{j} \phi_{r}(j) .
$$

Since $\phi_{0}(j)=1$ for all $j$, Lemma 6.2 implies that

$$
\sum_{j=0}^{n} L_{t}(i, j)=v_{0} \sum_{r=0}^{n} \frac{\phi_{r}(i) \phi_{r}(t)}{\left(v_{r}\right)^{2}} \delta_{r, 0}=1 .
$$


For the reversibility assertion, it is clear from the definition of $L_{t}$ that

$$
\frac{\mu_{i}}{|X|} L_{t}(i, j)=\frac{\mu_{j}}{|X|} L_{t}(j, i)
$$

for all $i, j$.

One uses the chain $L_{t}$ to construct an exchangeable pair $\left(W, W^{\prime}\right)$ in the usual way. First choose $i$ from the Plancherel measure, then choose $j$ with probability $L_{t}(i, j)$, and finally let $\left(W, W^{\prime}\right)=(W(i), W(j))$.

Lemma 6.6. $\mathbb{E}\left(W^{\prime} \mid W\right)=\left(\frac{\phi_{s}(t)}{v_{s}}\right) W$.

Proof. By the definitions and Lemma 6.2 one has that

$$
\begin{aligned}
\mathbb{E}\left(W^{\prime} \mid i\right) & =\frac{1}{\sqrt{v_{s}}} \sum_{j=0}^{n} L_{t}(i, j) \phi_{s}(j) \\
& =\frac{1}{\sqrt{v_{s}}} \frac{1}{|X|} \sum_{r=0}^{n} \frac{\phi_{r}(i) \phi_{r}(t)}{\left(v_{r}\right)^{2}} \sum_{j=0}^{n} \mu_{j} \phi_{s}(j) \phi_{r}(j) \\
& =\left(\frac{\phi_{s}(t)}{v_{s}}\right) W(i) .
\end{aligned}
$$

The result follows since this depends on $i$ only through $W$.

Corollary 6.7 will not be used but is worth recording.

Corollary 6.7. The eigenvalues of $L_{t}$ are $\frac{\phi_{s}(t)}{v_{s}}$ for $0 \leq s \leq n$. The functions $\psi_{s}(i)=\frac{\phi_{s}(i)}{\sqrt{v_{s}}}$ are a basis of eigenvectors of $L_{t}$, orthonormal with respect to the inner product

$$
\left\langle f_{1}, f_{2}\right\rangle=\sum_{i=0}^{n} f_{1}(i) \overline{f_{2}(i)} \frac{\mu_{i}}{|X|} .
$$

Proof. The proof of Lemma 6.6 shows that $\psi_{s}$ is an eigenvector of $L_{t}$ with eigenvalue $\frac{\phi_{s}(t)}{v}$. The orthonormality assertion follows from Lemma 6.2 and the basis assertion follows since $n+1$ linearly independent eigenvectors have been given.

Lemma 6.8. $\mathbb{E}\left(W^{\prime}-W\right)^{2}=2\left(1-\frac{\phi_{s}(t)}{v_{s}}\right)$.

Proof. This is immediate from Lemmas 2.3 and 6.6.

The next lemmas are quite helpful.

Lemma 6.9.

$$
\mathbb{E}\left[\left(W^{\prime}-W\right)^{2} \mid i\right]=v_{s} \sum_{r=0}^{n} p_{2}(r)\left(\frac{\phi_{r}(t)}{v_{r}}+1-\frac{2 \phi_{s}(t)}{v_{s}}\right) \frac{\phi_{r}(i)}{v_{r}} .
$$

$$
\operatorname{Var}\left(\mathbb{E}\left[\left(W^{\prime}-W\right)^{2} \mid i\right]\right)=\left(v_{s}\right)^{2} \sum_{r=1}^{n} \frac{p_{2}(r)^{2}}{v_{r}}\left(\frac{\phi_{r}(t)}{v_{r}}+1-\frac{2 \phi_{s}(t)}{v_{s}}\right)^{2} .
$$

Note that the sum in the second part of the lemma is over non-zero $r$. 
Proof. First observe that

$$
\begin{aligned}
\mathbb{E}\left(\left(W^{\prime}\right)^{2} \mid i\right) & =\frac{1}{v_{s}} \sum_{j=0}^{n} \frac{\mu_{j}}{|X|} \sum_{r=0}^{n} \frac{\phi_{r}(i) \phi_{r}(t) \phi_{r}(j)}{\left(v_{r}\right)^{2}} \phi_{s}(j)^{2} \\
& =\frac{1}{v_{s}} \sum_{r=0}^{n} \frac{\phi_{r}(i) \phi_{r}(t)}{\left(v_{r}\right)^{2}} \sum_{j=0}^{n} \frac{\mu_{j}}{|X|} \phi_{s}(j)^{2} \phi_{r}(j) \\
& =v_{s} \sum_{r=0}^{n} \frac{\phi_{r}(i) \phi_{r}(t)}{\left(v_{r}\right)^{2}} p_{2}(r) .
\end{aligned}
$$

The second equality switched the order of summation, and the third equality was Lemma 6.3 .

Next we claim that $W^{2}=v_{s} \sum_{r=0}^{n} p_{2}(r) \frac{\phi_{r}(i)}{v_{r}}$. To see this note that Lemma 6.3 gives

$$
\frac{1}{\left(v_{s}\right)^{2}} \sum_{j=0}^{n} \frac{\mu_{j}}{|X|} \phi_{s}(j)^{2} \phi_{r}(j)=p_{2}(r) .
$$

Then multiply both sides of the equation by $v_{s} \frac{\phi_{r}(i)}{v_{r}}$, sum over $r$, and apply Lemma 6.1 .

Now note from Lemma 6.6 that

$$
\begin{aligned}
\mathbb{E}\left[\left(W^{\prime}-W\right)^{2} \mid i\right] & =\mathbb{E}\left(\left(W^{\prime}\right)^{2} \mid i\right)-2 W \mathbb{E}\left(W^{\prime} \mid i\right)+W^{2} \\
& =\mathbb{E}\left(\left(W^{\prime}\right)^{2} \mid i\right)+\left(1-\frac{2 \phi_{s}(t)}{v_{s}}\right) W^{2}
\end{aligned}
$$

The first part of the lemma follows from this and the previous two paragraphs.

From the first part of the lemma and Lemma 6.2. one has that

$$
\mathbb{E}\left(\mathbb{E}\left[\left(W^{\prime}-W\right)^{2} \mid i\right]^{2}\right)=\left(v_{s}\right)^{2} \sum_{r=0}^{n} \frac{p_{2}(r)^{2}}{v_{r}}\left(\frac{\phi_{r}(t)}{v_{r}}+1-\frac{2 \phi_{s}(t)}{v_{s}}\right)^{2} .
$$

Since $p_{2}(0)=\frac{1}{v_{s}}$, it follows that the contribution to the above expression coming from $r=0$ is $4\left(1-\frac{\phi_{s}(t)}{v_{s}}\right)^{2}$. The second part of the lemma follows since by Lemma 6.8 .

$$
\operatorname{Var}\left(\mathbb{E}\left[\left(W^{\prime}-W\right)^{2} \mid i\right]\right)=\mathbb{E}\left(\mathbb{E}\left[\left(W^{\prime}-W\right)^{2} \mid i\right]^{2}\right)-4\left(1-\frac{\phi_{s}(t)}{v_{s}}\right)^{2}
$$

Lemma 6.10. Let $k$ be a positive integer.

(1) $\mathbb{E}\left(W^{\prime}-W\right)^{k}=\left(v_{s}\right)^{k / 2} \sum_{m=0}^{k}(-1)^{k-m}\left(\begin{array}{l}k \\ m\end{array}\right) \sum_{r=0}^{n} \frac{\phi_{r}(t)}{v_{r}} \frac{p_{m}(r) p_{k-m}(r)}{v_{r}}$.

(2) $\mathbb{E}\left(W^{\prime}-W\right)^{4}=v_{s}^{2}\left[\sum_{r=0}^{n}\left(8\left(1-\frac{\phi_{s}(t)}{v_{s}}\right)-6\left(1-\frac{\phi_{r}(t)}{v_{r}}\right)\right) \frac{p_{2}(r)^{2}}{v_{r}}\right]$. 
Proof. For the first assertion, note that $\mathbb{E}\left(\left(W^{\prime}-W\right)^{k} \mid i\right)$ is equal to

$$
\begin{aligned}
& \frac{1}{\left(v_{s}\right)^{k / 2}} \sum_{j=0}^{n} \frac{\mu_{j}}{|X|} \sum_{r=0}^{n} \frac{\phi_{r}(i) \phi_{r}(t) \phi_{r}(j)}{\left(v_{r}\right)^{2}}\left(\phi_{s}(j)-\phi_{s}(i)\right)^{k} \\
= & \frac{1}{\left(v_{s}\right)^{k / 2}} \sum_{m=0}^{k}(-1)^{k-m}\left(\begin{array}{c}
k \\
m
\end{array}\right) \phi_{s}(i)^{k-m} \sum_{r=0}^{n} \frac{\phi_{r}(i) \phi_{r}(t)}{\left(v_{r}\right)^{2}} \\
& \cdot \sum_{j=0}^{n} \frac{\mu_{j}}{|X|} \phi_{s}(j)^{m} \phi_{r}(j) \\
= & \frac{1}{\left(v_{s}\right)^{k / 2}} \sum_{m=0}^{k}(-1)^{k-m}\left(\begin{array}{c}
k \\
m
\end{array}\right) \phi_{s}(i)^{k-m}\left(v_{s}\right)^{m} \sum_{r=0}^{n} \frac{\phi_{r}(i) \phi_{r}(t) p_{m}(r)}{\left(v_{r}\right)^{2}}
\end{aligned}
$$

where the last equality is Lemma 6.3. Consequently,

$$
\begin{aligned}
\mathbb{E}\left(W^{\prime}-W\right)^{k}= & \mathbb{E}\left(\mathbb{E}\left(\left(W^{\prime}-W\right)^{k} \mid i\right)\right) \\
= & \left(v_{s}\right)^{k / 2} \sum_{m=0}^{k}(-1)^{k-m}\left(\begin{array}{c}
k \\
m
\end{array}\right) \\
& \cdot \sum_{r=0}^{n} \frac{\phi_{r}(t)}{\left(v_{r}\right)^{2}} p_{m}(r) \sum_{i=0}^{n} \frac{\mu_{i}}{|X|} \frac{\phi_{s}(i)^{k-m} \phi_{r}(i)}{\left(v_{s}\right)^{k-m}} .
\end{aligned}
$$

The first assertion now follows from Lemma 6.3.

For the second part, the first assertion gives that

$$
\mathbb{E}\left(W^{\prime}-W\right)^{4}=\left(v_{s}\right)^{2} \sum_{m=0}^{4}(-1)^{m}\left(\begin{array}{c}
4 \\
m
\end{array}\right) \sum_{r=0}^{n} \frac{\phi_{r}(t)}{v_{r}} \frac{p_{m}(r) p_{4-m}(r)}{v_{r}} .
$$

By page 654 of [30], $\phi_{r}(0)=v_{r}$ for all $r$. Thus specializing to $t=0$ shows that

$$
0=\left(v_{s}\right)^{2} \sum_{m=0}^{4}(-1)^{m}\left(\begin{array}{c}
4 \\
m
\end{array}\right) \sum_{r=0}^{n} \frac{p_{m}(r) p_{4-m}(r)}{v_{r}} .
$$

So for general $t$, one has that

$$
\mathbb{E}\left(W^{\prime}-W\right)^{4}=-\left(v_{s}\right)^{2} \sum_{m=0}^{4}(-1)^{m}\left(\begin{array}{c}
4 \\
m
\end{array}\right) \sum_{r=0}^{n}\left(1-\frac{\phi_{r}(t)}{v_{r}}\right) \frac{p_{m}(r) p_{4-m}(r)}{v_{r}} .
$$

The contribution from the $m=0,4$ terms is 0 , since $p_{0}(r)=0$ if $r \neq 0$. The contribution from the $m=2$ term is

$$
-6\left(v_{s}\right)^{2} \sum_{r=0}^{n}\left(1-\frac{\phi_{r}(t)}{v_{r}}\right) \frac{p_{2}(r)^{2}}{v_{r}}
$$

The $m=1,3$ terms are equal and together contribute

$$
\begin{aligned}
8\left(v_{s}\right)^{2} \sum_{r=0}^{n}\left(1-\frac{\phi_{r}(t)}{v_{r}}\right) \frac{p_{1}(r) p_{3}(r)}{v_{r}} & =8\left(v_{s}\right)^{2}\left(1-\frac{\phi_{s}(t)}{v_{s}}\right) \frac{p_{3}(s)}{v_{s}} \\
& =8\left(v_{s}\right)^{2}\left(1-\frac{\phi_{s}(t)}{v_{s}}\right) \sum_{r=0}^{n} \frac{p_{2}(r)^{2}}{v_{r}}
\end{aligned}
$$

where the final equality is part (2) of Lemma 6.4. Adding the terms completes the proof of the second assertion. 
Arguing as in the proof of Theorem 3.12 and using the above lemmas, one obtains the following result.

Theorem 6.11. Fix $s \in\{0, \cdots, n\}$, and let $W=\frac{\phi_{s}(i)}{\sqrt{v_{s}}}$ where $i$ is chosen from the Plancherel measure of the association scheme. Fix $t$ such that $0<\frac{\phi_{s}(t)}{v_{s}}<1$. Then for all real $x_{0}$,

$$
\begin{gathered}
\left|\mathbb{P}\left(W \leq x_{0}\right)-\frac{1}{\sqrt{2 \pi}} \int_{-\infty}^{x_{0}} e^{-\frac{x^{2}}{2}} d x\right| \\
\leq \frac{v_{s}}{a} \sqrt{\sum_{r=1}^{n} \frac{p_{2}(r)^{2}}{v_{r}}\left(\frac{\phi_{r}(t)}{v_{r}}+1-\frac{2 \phi_{s}(t)}{v_{s}}\right)^{2}} \\
+\frac{\sqrt{v_{s}}}{(\pi)^{1 / 4}}\left[\sum_{r=0}^{n}\left(8-\frac{6}{a}\left(1-\frac{\phi_{r}(t)}{v_{r}}\right)\right) \frac{p_{2}(r)^{2}}{v_{r}}\right]^{1 / 4},
\end{gathered}
$$

where $a=1-\frac{\phi_{s}(t)}{v_{s}}$.

6.3. Example: Hamming scheme. This subsection illustrates the theory of Subsection 6.2 for the Hamming scheme $H(d, q)$, where $d, q \geq 2$ are positive integers.

To begin we recall the definition of $H(d, q)$ and its basic properties, referring the reader to Chapter 3 of 2 for more details. The elements $X$ of $H(d, q)$ are $d$-tuples of numbers chosen from $\{1, \cdots, q\}$; clearly $|X|=q^{d}$. A pair $(x, y)$ is in $R_{i}$ if $x$ and $y$ differ in exactly $i$ coordinates. For $0 \leq i \leq d$, one has that $v_{i}=(q-1)^{i}\left(\begin{array}{l}d \\ i\end{array}\right)$ and $\mu_{i}=(q-1)^{i}\left(\begin{array}{c}d \\ i\end{array}\right)$. Thus the Plancherel measure on $\{0, \cdots, d\}$ chooses $i$ with probability $\frac{(q-1)^{i}\left(\begin{array}{l}d \\ i\end{array}\right)}{q^{d}}$. The numbers $\phi_{s}(i)$ are equal to

$$
\sum_{j=0}^{s}(-1)^{j}(q-1)^{s-j}\left(\begin{array}{l}
i \\
j
\end{array}\right)\left(\begin{array}{l}
d-i \\
s-j
\end{array}\right) .
$$

The polynomial $\phi_{s}$ in the variable $i$ is known as a $q$-Krawtchouk polynomial.

In what follows $W=\frac{\phi_{1}(i)}{\sqrt{v_{1}}}$, where $i$ is chosen from the Plancherel measure of the Hamming scheme $H(d, q)$. The Hamming graph has vertex set $X$ and an edge between two vertices if they differ in one coordinate. Thus the eigenvalues of the adjacency matrix of the Hamming graph are $\phi_{1}(i)$ with multiplicity $\mu_{i}$, which motivates the study of $W$. Hora [22] shows that if $d \rightarrow \infty$ and $q / d \rightarrow 0$, then $W$ converges in distribution to a normal random variable with mean 0 and variance 1 .

In fact since $W(i)=\frac{(q-1) d-q i}{\sqrt{(q-1) d}}$ and $\frac{\mu_{i}}{|X|}=\frac{(q-1)^{i}\left(\begin{array}{l}d \\ i\end{array}\right)}{q^{d}}$, it is straightforward that $W$ has the same distribution as

$$
\frac{Y_{1}+\cdots+Y_{d}}{\sqrt{(q-1) d}}
$$

where the $Y$ 's are independent random variables, each equal to $q-1$ with probability $\frac{1}{q}$ and to -1 with probability $1-\frac{1}{q}$. Hence the Berry-Esseen theorem [11] shows that $W$ satisfies a central limit theorem with the error term $C \sqrt{\frac{q}{d}}$ where $C$ is a small explicit constant.

For comparison, let us study $W$ using Theorem 6.11 with $s=1$ and $t=1$. Then $a=\frac{q}{(q-1) d}$ and from the probabilistic interpretation of $p_{m}(r)$ one computes that $p_{2}(0)=\frac{1}{(q-1) d}, p_{2}(1)=\frac{(q-2)}{(q-1) d}$, and $p_{2}(2)=1-\frac{1}{d}$. The first term in the error term 
of Theorem 6.11 is then computed to equal $\sqrt{\frac{(q-2)^{2}}{(q-1) d}}$, which is less than $\sqrt{\frac{q}{d}}$. The second term in the error term of Theorem 6.11 is computed to equal $\left(\frac{2 q^{2}}{\pi(q-1) d}\right)^{1 / 4}$, which is at most $\left(\frac{2 q}{d}\right)^{1 / 4}$. Thus one obtains a central limit theorem for $W$ with error term $\sqrt{\frac{q}{d}}+\left(\frac{2 q}{d}\right)^{1 / 4}$.

To close this section, it is shown how our exchangeable pair can be used to give $O\left(d^{-1 / 2}\right)$ bounds for $q$ fixed. The key is Lemma 6.12, which shows that the Markov chain $L_{1}$ is actually a birth-death chain.

Lemma 6.12. The Markov chain $L_{1}$ on the set $\{0, \cdots, d\}$ is a birth-death chain with transition probabilities

$$
L_{1}(i, j)= \begin{cases}\frac{i}{d(q-1)} & \text { if } j=i-1 \\ \frac{i}{d}\left(1-\frac{1}{q-1}\right) & \text { if } j=i . \\ 1-\frac{i}{d} & \text { if } j=i+1\end{cases}
$$

Proof. Recall that $L_{1}(i, j)=\frac{\mu_{j}}{|X|} \sum_{r=0}^{n} \frac{\phi_{r}(i) \phi_{r}(1) \phi_{r}(j)}{\left(v_{r}\right)^{2}}$. From the formula for $\phi_{r}(l)$ one sees that

$$
\phi_{r}(l)=(q-1)^{r-l} \frac{\left(\begin{array}{l}
d \\
r
\end{array}\right)}{\left(\begin{array}{l}
d \\
l
\end{array}\right)} \phi_{l}(r)
$$

for any $0 \leq r, l \leq d$. Thus

$$
L_{1}(i, j)=\frac{\mu_{j}}{|X| d\left(\begin{array}{c}
d \\
i
\end{array}\right)\left(\begin{array}{l}
d \\
j
\end{array}\right)(q-1)^{i+j+1}} \sum_{r=0}^{n}\left(\begin{array}{l}
d \\
r
\end{array}\right)(q-1)^{r} \phi_{1}(r) \phi_{i}(r) \phi_{j}(r) .
$$

From page 152 of [30], there is a recurrence relation

$$
(i+1) \phi_{i+1}(r)=[(d-i)(q-1)+i-q r] \phi_{i}(r)-(q-1)(d-i+1) \phi_{i-1}(r) .
$$

Since $\phi_{1}(r)=d(q-1)-q r$, the recurrence is equivalent to

$$
\phi_{1}(r) \phi_{i}(r)=i(q-2) \phi_{i}(r)+(i+1) \phi_{i+1}(r)+(q-1)(d-i+1) \phi_{i-1}(r) .
$$

Applying this to the expression for $L_{1}(i, j)$ at the end of the previous paragraph, the result follows from Lemma 6.2 .

Lemma 6.12 implies that $\left|W^{\prime}-W\right| \leq A$ with $A=\frac{q}{\sqrt{(q-1) d}}$. Thus one can apply the version of Theorem 6.11 which would arise by using Theorem 2.2 instead of Theorem 2.1. Recalling that $a=\frac{q}{(q-1) d}$, one obtains an error term of $\frac{1}{2} \sqrt{\frac{q}{d}}+$ $\frac{.41 q^{2}+1.5 q}{\sqrt{(q-1) d}}$. For $q$ fixed this goes as $d^{-1 / 2}$.

\section{REFERENCES}

1. Aldous, D. and Diaconis, P., Longest increasing subsequences: from patience sorting to the Baik-Deift-Johansson theorem, Bull. Amer. Math. Soc. 36 (1999), 413-432. MR1694204 (2000g:60013)

2. Bannai, E. and Ito, T., Algebraic combinatorics, I. Association schemes, Benjamin/Cummings Publishing Co., Inc., Menlo Park, CA, 1984. MR882540 (87m:05001)

3. Borodin, A., Okounkov, A., and Olshanski, G., Asymptotics of Plancherel measure for symmetric groups, J. Amer. Math. Soc. 13 (2000), 481-515. MR1758751 (2001g:05103)

4. Borodin, A. and Olshanski, G., Z-measures on partitions and their scaling limits, European J. Combin. 26 (2005), 795-834. MR2143199 (2006d:60018) 
5. Borodin, A. and Olshanski, G., Harmonic functions on multiplicative graphs and interpolation polynomials, Elecron. J. Combin. 7 (2000), Research paper 28, 39 pages. MR.1758654 (2001f:05160)

6. Chatterjee, S. and Fulman, J., Exponential approximation by exchangeable pairs and spectral graph theory, (2006), preprint math.PR/0605552 at http://xxx.lanl.gov.

7. Deift, P., Integrable systems and combinatorial theory, Notices Amer. Math. Soc. 47 (2000), 631-640. MR1764262 (2001g:05012)

8. Diaconis, P., Group representations in probability and statistics, Institute of Mathematical Statistics Lecture Notes, Volume 11, 1988. MR964069 (90a:60001)

9. Diaconis, P. and Holmes, S., Random walks on trees and matchings, Elec. J. Probab. 7 (2002), 17 pages (electronic). MR $1887626(2002 \mathrm{k}: 60025)$

10. Diaconis, P. and Shahshahani, M., Generating a random permutation with random transpositions, Z. Wahr. Verw. Gebiete 57 (1981), 159-179. MR626813 (82h:60024)

11. Durrett, R., Probability: Theory and examples, Second edition, Duxbury Press, Belmont, CA, 1996. MR 1609153 (98m:60001)

12. Eskin, A. and Okounkov, A., Asymptotics of branched coverings of a torus and volumes of moduli spaces of holomorphic differentials, Invent. Math. 145 (2001), 59-103. MR1839286 (2002g:32018)

13. Eymard, P. and Roynette, B., Marches aléatoires sur le dual de SU(2), in Analyse harmonique sur les groupes de Lie, Springer Lecture Notes in Math, Volume 497 (1975), 108-152. MR0423457 (54:11435)

14. Fulman, J., Stein's method and Plancherel measure of the symmetric group, Transac. Amer. Math. Soc. 357 (2005), 555-570. MR2095623 (2005e:05156)

15. Fulman, J., Stein's method, Jack measure, and the Metropolis algorithm, J. Combin. Theory Ser. A 108 (2004), 275-296. MR2098845 (2005h:60021)

16. Fulman, J., An inductive proof of the Berry-Esseen theorem for character ratios, Ann. Combin. 10 (2006), 319-332. MR2284273 (2007i:05189)

17. Fulman, J., Martingales and character ratios, Trans. Amer. Math. Soc. 358 (2006), 4533-4552. MR 2231387 (2007e:05178)

18. Gross, B., Some applications of Gelfand pairs to number theory, Bull. Amer. Math. Soc. 24 (1991), 277-301. MR1074028 (91i:11055)

19. Hanlon, P., Stanley, R., and Stembridge, J., Some combinatorial aspects of the spectra of normally distributed random matrices, in Hypergeometric functions on domains of positivity, Jack polynomials, and applications, Contemporary Mathematics, Volume 138 (1992), 151-174. MR 1199126 (93j:05164)

20. Hoffman, P. and Humphreys, J., Projective representations of the symmetric group, Oxford University Press, New York, 1992. MR.1205350 (94f:20027)

21. Hora, A., Central limit theorem for the adjacency operators on the infinite symmetric group, Comm. Math. Phys. 195 (1998), 405-416. MR1637801(99i:46058)

22. Hora, A., Central limit theorems and asymptotic spectral analysis in large graphs, Infin. Dimens. Anal. Quantum Probab. Relat. Top. 1 (1998), 221-246. MR1628244 (99g:46089)

23. Ivanov, V., The Gaussian limit for projective characters of large symmetric groups, J. Math. Sci. (N.Y.), 121 (2004), 2330-2344. Translated from Zap. Nauchn. Sem. POMI 283 (2001), 73-97. MR 1879064 (2002k:60061)

24. Ivanov, V. and Olshanski, G., Kerov's central limit theorem for the Plancherel measure on Young diagrams, in Symmetric Functions 2001: Surveys of developments and perspectives, Kluwer Academic Publishers, Dodrecht, 2002.

25. Johansson, K., Discrete orthogonal polynomial ensembles and the Plancherel measure, Ann. of Math. 153 (2001), 259-296. MR.1826414 (2002g:05188)

26. Kerov, S.V., Gaussian limit for the Plancherel measure of the symmetric group, Compt. Rend. Acad. Sci. Paris, Serie I 316 (1993), 303-308. MR1204294 (93k:20106)

27. Kerov, S.V., Anisotropic Young diagrams and Jack symmetric functions, Funct. Anal. Appl. 34 (2000), 41-51. MR1756734 (2001f:05158)

28. Letac, G., Les fonctions sphériques d'un couple de Gelfand symétrique et les chaînes de Markov, Adv. Appl. Probab. 14 (1982), 272-294. MR650123 (83i:60078)

29. Macdonald, I., Symmetric functions and Hall polynomials, Second edition, Oxford University Press, New York, 1995. MR.1354144 (96h:05207) 
30. Macwilliams, F. and Sloane, N., The theory of error-correcting codes, Elsevier Science B.V., Amsterdam, 1977.

31. Matsumoto, S., Correlation functions of the shifted Schur measure, J. Math. Soc. Japan 57 (2005), 619-637. MR2139724 (2006a:60013)

32. Okounkov, A., Random matrices and random permutations, Internat. Math. Res. Notices 20 (2000), 1043-1095. MR1802530(2002c:15045)

33. Okounkov, A., The uses of random partitions, XIVth International Congress on Mathematical Physics, 379-403, World Sci. Publ., Hackensack, NJ 2005. MR2227852 (2007c:05013)

34. Reinert, G., Couplings for normal approximations with Stein's method, in Microsurveys in discrete probability, DIMACS Ser. Discrete Math. Theoret. Comput. Sci., Volume 41 (1998), 193-207. MR:1630415 (99h:60043)

35. Rinott, Y. and Rotar, V., On coupling constructions and rates in the CLT for dependent summands with applications to the antivoter model and weighted U-statistics, Ann. of Appl. Probab. 7 (1997), 1080-1105. MR1484798 (99g:60050)

36. Rinott, Y. and Rotar, V., Normal approximations by Stein's method, Decis. Econ. Finance 23 (2000), 15-29. MR.1780090 (2001h:60037)

37. Sagan, B., The symmetric group. Representations, combinatorial algorithms, and symmetric functions, Second edition, Springer-Verlag, New York, 2001. MR.1824028 (2001m:05261)

38. Serre, J-P., Linear representations of finite groups, Springer-Verlag, New York, 1977. MR0450380 (56:8675)

39. Shao, Q., and Su, Z., The Berry-Esseen bound for character ratios, Proc. Amer. Math. Soc. 134 (2006), 2153-2159. MR2215787

40. Sniady, P., Gaussian fluctuations of characters of symmetric groups and of Young diagrams, Probab. Theory Related Fields 136 (2006), 263-297. MR2240789 (2007d:20020)

41. Stein, C., Approximate computation of expectations, Institute of Mathematical Statistics Lecture Notes, Volume 7, 1986. MR882007 (88j:60055)

42. Stembridge, J., On Schur's Q-functions and the primitive idempotents of a commutative Hecke algebra, J. Algebraic Combin. 1 (1992), 71-95. MR1162642 (93c:05113)

43. Terras, A., Fourier analysis on finite groups and applications, London Math. Society Student Texts 43, Cambridge University Press, Cambridge, 1999. MR.1695775 (2000d:11003)

44. Terras, A., Survey of spectra of Laplacians on finite symmetric spaces, Experiment. Math. 5 (1996), 15-32. MR.1412951 (97m:11154)

45. Tracy, C. and Widom, H., A limit theorem for shifted Schur measures, Duke Math. Journal 123 (2004), 171-208. MR2060026 (2006c:60028)

Department of Mathematics, University of Pittsburgh, Pittsburgh, Pennsylvania 15260

Current address: Department of Mathematics, University of Southern California, Los Angeles, California 90089-2532

E-mail address: fulman@usc.edu 